\title{
A circle criterion for strong integral input-to-state stability
}

\author{
Chris Guiver*† Hartmut Logemann*
}

June 12, 2019

\begin{abstract}
We present sufficient conditions for integral input-to-state stability (iISS) and strong iISS of the zero equilibrium pair of continuous-time forced Lur'e systems, where by strong iISS we mean the conjunction of iISS and small-signal ISS. Our main results are reminiscent of the complex Aizerman conjecture and the well-known circle criterion. We derive a number of corollaries, including a result on stabilisation by static feedback in the presence of input saturation. In particular, we identify classes of forced Lur'e systems with saturating nonlinearities which are strongly iISS, but not ISS.
\end{abstract}

Keywords. Asymptotic stabilization, circle criterion, Lur'e systems, robust control of nonlinear systems, strong iISS

MSC(2010). 93C10, 93C35, 93C80, 93D05, 93D09, 93D10, 93D15, 93D20, 93D25.

\section{Introduction}

We study (strong) integral input-to-state stability properties of the class of forced Lur'e systems:

$$
\dot{x}=A x+B f\left(C x+D_{\mathrm{e}} v\right)+B_{\mathrm{e}} v, \quad x(0)=x^{0} .
$$

Here $A, B, B_{\mathrm{e}}, C$ and $D_{\mathrm{e}}$ are real matrices, $f$ is a (nonlinear) function, $x$ denotes the state, with initial state $x^{0}$, and $v$ is a forcing function (also named, or interpreted, as a disturbance, control or input). Lur'e systems are a common and important class of nonlinear control system, and arise in a number of engineering scenarios, such as the stabilisation of linear systems by saturated static feedback. The study of the stability properties of Lur'e systems constitutes absolute stability theory which, loosely speaking, seeks to conclude stability of the feedback system (1.1) through the interplay of frequency domain properties of the linear system given by $(A, B, C)$ and boundedness or sector properties of the nonlinearity $f,[22,41]$. Recently, absolute stability ideas have been merged with inputto-state stability (ISS) theory to obtain ISS criteria which resemble classical absolute stability results, see $[2,11,19,20,29,30,31]$. The ISS criteria obtained in [29] have been used in [5] to prove converging-input converging-state properties for Lur'e systems.

The concepts of ISS and integral ISS (iISS) were introduced in [35] and [36], respectively. Over the last

\footnotetext{
*Department of Mathematical Sciences, University of Bath, Bath, UK, c.guiver@bath.ac.uk,h.logemann@bath.ac.uk

${ }^{\dagger}$ Corresponding author

Submitted: June 2018, revised: January and June 2019
}

30 years, an extensive ISS Lyapunov theory has been developed, synthesizing state-space and input-output viewpoints. The result is a comprehensive stability theory for nonlinear control systems see, for example, the survey papers [8, 37]. Roughly speaking, ISS and iISS mean that the state has "nice" boundedness properties, expressed in terms of suitable comparison functions, with respect to the norm of initial states and the "size" of the input signals. In the case of ISS, the "size" of the input signal is its $L^{\infty}$-norm, whilst the integral of the norm of the input plays a key role in the context of iISS. ISS implies iISS, but the converse is false in general.

Strong iISS is a recent stability concept, introduced in [6], and is the conjunction of iISS and ISS with respect to small signals, or small-signal ISS. The smallsignal ISS property guarantees that the boundedness of the state is robust with respect to small, but potentially persistent, forcing. The emphasis of the current paper is on strong iISS, which is an "intermediate property" with the benefits of "the robustness strengths of ISS and the generality of iISS" [6].

The results obtained in this paper are reminiscent of the complex Aizerman conjecture [16, 17] and the circle criterion $[14,20,22]$ : we show that, when suitably modified, these classical absolute stability results are sufficient for (strong) iISS. In particular, our main result, Theorem 3.1, shows that if every complex output feedback gain matrix in the open ball $\mathbb{B}_{\mathbb{C}}(K, r)$ (centred at $K$ and of radius $r>0$ ) stabilises the underlying linear system $(A, B, C)$, then the zero equilibrium pair of the forced Lur'e system (1.1) is strongly iISS for all $f$ satisfying the inequality

$$
\|f(z)-K z\| \leq r\|z\|-\alpha(\|z\|) \quad \forall z \in \mathbb{R}^{p},
$$

where $\alpha: \mathbb{R}_{+} \rightarrow \mathbb{R}_{+}$is a comparison function of class $\mathcal{K}$ (see Section 2 for the definition of $\mathcal{K}$ ).

The contribution of this work very much resonates with that in the paper [29], where ISS conditions for Lur'e systems are derived which are inspired by the complex Aizerman conjecture and the circle criterion. In particular, the condition (1.2) plays an important role in [29]. Not surprisingly, the sense in which (1.2) holds, that is, for which types of $\alpha$, is crucial for the stability property which may be inferred, see the discussion in Section 5, in particular Theorem 5.1. The consequences of Theorem 3.1 include a strong iISS version of the well-known circle criterion which provides a sufficient condition for strong iISS in terms of positive- 
real and sector properties of the transfer function and the nonlinearity, respectively, see Corollary 3.8 .

One motivation for the present work is its relevance to the stabilisation of linear systems subject to input saturation, a problem which has received considerable and persistent attention in the literature, see, for instance $[3,9,12,23,24,32,33,34,38,39]$. It is wellknown that a necessary condition for stabilisation (in the sense of 0-GAS) is that $A$ has no eigenvalues with positive real part and that the pair $(A, B)$ is stabilisable. More recently, it was shown in [29, Proposition 3.4] that if $A$ has eigenvalues with non-negative real part, then the zero equilibrium pair of (1.1) system cannot be ISS when $f$ is bounded (in particular, when $f$ is a saturation nonlinearity). In Theorem 4.2, we provide sufficient conditions for the zero equilibrium pair of such a feedback interconnection to be strongly iISS for a class of saturating nonlinearities. Theorem 4.2 strengthens the recent result [3, Theorem 2] which considers the same problem. We discuss how our results relate to others in the literature in Remark 4.5.

The paper is organised as follows. Section 2 gathers notation and preliminaries. Our main results are presented in Section 3, and are applied to a class of Lur'e systems with saturating nonlinearities in Section 4. Section 5 contains a discussion which places the our work in the wider context provided by related papers in the literature. To avoid disruption to the presentation, all proofs appear in the appendices.

\section{Notation and preliminaries}

The set of positive integers is denoted by $\mathbb{N}$, and $\mathbb{R}$ and $\mathbb{C}$ denote the fields of real and complex numbers, respectively. We set $\mathbb{R}_{+}:=\{r \in \mathbb{R}: r \geq 0\}$ and $\mathbb{N}_{0}=\mathbb{N} \cup\{0\}$. For $n \in \mathbb{N}, \mathbb{R}^{n}$ and $\mathbb{C}^{n}$ denote the usual real and complex $n$-dimensional vector spaces, respectively, both equipped with the 2-norm denoted by $\|\cdot\|$ induced by the standard inner product $\langle\cdot, \cdot\rangle$.

For $m \in \mathbb{N}$, let $\mathbb{R}^{n \times m}$ and $\mathbb{C}^{n \times m}$ denote the normed linear spaces of $n \times m$ matrices with real and complex entries, respectively, both equipped with the operator norm induced by the 2-norm, also denoted by $\|\cdot\|$. A matrix $M \in \mathbb{C}^{n \times n}$ is said to be Hurwitz if all its eigenvalues have negative real parts.

For $K \in \mathbb{R}^{m \times p}, \mathbb{F}=\mathbb{R}$ or $\mathbb{F}=\mathbb{C}$, and $r>0$, we set

$$
\mathbb{B}_{\mathbb{F}}(K, r):=\left\{Z \in \mathbb{F}^{m \times p}:\|Z-K\|<r\right\},
$$

the open ball in $\mathbb{F}^{m \times p}$ centred at $K$ and of radius $r$.

We recall terminology and definitions pertaining to so-called comparison functions. Let $\mathcal{K}$ denote the set of all continuous and strictly increasing functions $\phi$ : $\mathbb{R}_{+} \rightarrow \mathbb{R}_{+}$such that $\phi(0)=0$. Note that if $\phi \in \mathcal{K}$, then $\phi(s)>0$ for all $s>0$. The subset of $\mathcal{K}$ consisting of all unbounded functions is denoted by $\mathcal{K}_{\infty}$. Obviously, if $\phi \in \mathcal{K}_{\infty}$, then $\phi(s) \rightarrow \infty$ as $s \rightarrow \infty$. The set $\mathcal{K} \mathcal{L}$ consists of all functions $\phi: \mathbb{R}_{+} \times \mathbb{R}_{+} \rightarrow \mathbb{R}_{+}$which satisfy $\phi(\cdot, t) \in \mathcal{K}$ for all $t \geq 0$ and, for all $s \geq 0, \phi(s, \cdot)$ is non-increasing with $\phi(s, t) \rightarrow 0$ as $t \rightarrow \infty$. Following the convention of [36], we do not impose continuity in the definition of a $\mathcal{K} \mathcal{L}$-function. By [36, Proposition 7 or Lemma 8], it follows that a discontinuous $\mathcal{K} \mathcal{L}$ function can be bounded from above by a continuous one. The reader is referred to [21] for more information on comparison functions.

As usual, $L^{1}\left(\mathbb{R}_{+}, \mathbb{R}^{n}\right)$ and $L^{\infty}\left(\mathbb{R}_{+}, \mathbb{R}^{n}\right)$ denote the spaces of (equivalence classes of) measurable functions $\mathbb{R}_{+} \rightarrow \mathbb{R}^{n}$ which are integrable and essentially bounded, respectively. The space of measurable locally essentially bounded functions $f: \mathbb{R}_{+} \rightarrow \mathbb{R}^{n}$ is denoted by $L_{\text {loc }}^{\infty}\left(\mathbb{R}_{+}, \mathbb{R}^{n}\right)$.

Consider the initial value problem (1.1). Here, and throughout,

$$
\begin{aligned}
\Sigma & :=\left(A, B, C, B_{\mathrm{e}}, D_{\mathrm{e}}\right) \\
& \in \mathbb{R}^{n \times n} \times \mathbb{R}^{n \times m} \times \mathbb{R}^{p \times n} \times \mathbb{R}^{n \times q} \times \mathbb{R}^{p \times q},
\end{aligned}
$$

for fixed $n, m, p, q \in \mathbb{N}$. Further, $f: \mathbb{R}^{p} \rightarrow \mathbb{R}^{m}$ in (1.1) is locally Lipschitz. For given $x^{0} \in \mathbb{R}^{n}$ and $v \in$ $L_{\text {loc }}^{\infty}\left(\mathbb{R}_{+}, \mathbb{R}^{q}\right)$, we let $x=x\left(\cdot ; x^{0}, v\right)$ denote the unique maximally defined absolutely continuous forward solution of the initial value problem (1.1). Note that if $f$ is affinely linearly bounded, that is, there exist positive constants $a$ and $b$ such that $\|f(z)\| \leq a+b\|z\|$ for all $z \in \mathbb{R}^{p}$, then (1.1) is forward complete, meaning that, for all $x^{0} \in \mathbb{R}^{n}$ and all $v \in L_{\mathrm{loc}}^{\infty}\left(\mathbb{R}_{+}, \mathbb{R}^{q}\right)$, the solution $x$ is defined on $\mathbb{R}_{+}$(see, for example, [26, Proposition $4.12])$.

We refer to (1.1) with $v=0$, as the unforced Lur'e system. If 0 is an equilibrium of the unforced system (1.1), then we abbreviate "global asymptotic stability of the zero equilibrium of the unforced system (1.1)" to the familiar "system (1.1) is 0-GAS".

The Lur'e system (1.1) may be seen as the closed-loop system arising from the feedback interconnection of the forced linear system

$$
\left.\begin{array}{l}
\dot{x}=A x+B u+B_{\mathrm{e}} v, \quad x(0)=x^{0} \\
y=C x+D_{\mathrm{e}} v
\end{array}\right\}
$$

with state $x$, input $u$, output $y$ and forcing $v$, and the static nonlinear output feedback

$$
u=f(y) .
$$

Note that (2.2) encompasses systems of the form

$$
\dot{x}=A x+B f(C x+d)+w, \quad x(0)=x^{0},
$$

where $d$ is an output disturbance and $w$ is another forcing function. Indeed, setting $q:=n+p$,

$$
B_{\mathrm{e}}:=\left(\begin{array}{ll}
I & 0
\end{array}\right), \quad D_{\mathrm{e}}:=\left(\begin{array}{ll}
0 & I
\end{array}\right), \text { and } v:=\left(\begin{array}{l}
w \\
d
\end{array}\right),
$$

it is clear that system (2.3) can be written in the form (2.2). Therefore, we focus attention on (2.2), or, equivalently, on (1.1). 
We call $\left(x^{*}, v^{*}\right) \in \mathbb{R}^{n} \times \mathbb{R}^{q}$ an equilibrium pair of (1.1) if $A x^{*}+B f\left(C x^{*}+D_{\mathrm{e}} v^{*}\right)+B_{\mathrm{e}} v^{*}=0$, that is, $x^{*}$ is a constant solution of (1.1) with constant forcing $v(t) \equiv v^{*}$. Without loss of generality, we shall assume throughout that $f(0)=0$ and $\left(x^{*}, v^{*}\right)=(0,0)-$ called the zero equilibrium pair. The general case can be reduced to the zero equilibrium pair.

Following [6], the zero equilibrium pair is said to be strongly iISS if it is iISS and small-signal ISS. The iISS property was introduced in [36], see also [1], and means that there exist $\beta \in \mathcal{K} \mathcal{L}$ and $\gamma_{1}, \gamma_{2} \in \mathcal{K}$ such that, for all $x^{0} \in \mathbb{R}^{n}$ and all $v \in L_{\mathrm{loc}}^{\infty}\left(\mathbb{R}_{+}, \mathbb{R}^{q}\right)$, and all $t \geq 0$

$$
\|x(t)\| \leq \beta\left(\left\|x^{0}\right\|, t\right)+\gamma_{1}\left(\int_{0}^{t} \gamma_{2}(\|v(s)\|) d s\right) .
$$

Furthermore, we say that the zero equilibrium pair is small-signal ISS if there exist $R>0, \beta \in \mathcal{K} \mathcal{L}$ and $\gamma \in \mathcal{K}$ such that: for all $x^{0} \in \mathbb{R}^{n}$, all $v \in L_{\text {loc }}^{\infty}\left(\mathbb{R}_{+}, \mathbb{R}^{q}\right)$, and all $t \geq 0$,

$$
\begin{aligned}
\|v\|_{L^{\infty}(0, t)} & <R \\
\Rightarrow \quad\|x(t)\| & \leq \beta\left(\left\|x^{0}\right\|, t\right)+\gamma\left(\|v\|_{L^{\infty}(0, t)}\right) .
\end{aligned}
$$

The $\mathcal{K}$-function $\gamma_{2}$ in $(2.4)$ is sometimes called an "iISS gain" for the system (1.1) The constant $R$ which appears in the definition of small-signal ISS is referred to as an input threshold [6].

In the following, for the sake of brevity, we shall say that (1.1) is strongly iISS if the zero equilibrium pair is strongly iISS. We adopt a similar convention for other stability notions. If (2.5) holds with $R=\infty$, then we say that (1.1) is ISS.

Remark 2.1. The following statements are consequences of routine arguments.

(i) If (1.1) is strongly iISS and $v \in L_{\text {loc }}^{\infty}\left(\mathbb{R}_{+}, \mathbb{R}^{q}\right)$ is such that $v(t) \rightarrow 0$ as $t \rightarrow \infty$, then $x\left(t ; x^{0}, v\right) \rightarrow 0$ as $t \rightarrow \infty$.

(ii) If (1.1) is iISS and $v \in L_{\mathrm{loc}}^{\infty}\left(\mathbb{R}_{+}, \mathbb{R}^{q}\right)$ is such that the function $t \mapsto \gamma_{2}(\|v(t)\|)$ is integrable, then $x\left(t ; x^{0}, v\right) \rightarrow 0$ as $t \rightarrow \infty$.

Throughout the present work, we let $\mathbf{G}$, given by $\mathbf{G}(s)=C(s I-A)^{-1} B$, denote the transfer function of the linear system specified by $(A, B, C)$ (that is, the transfer function of $(2.2 \mathrm{a})$ from input $u$ to output $y)$. Applying static output feedback to $(A, B, C)$ with gain $K \in \mathbb{R}^{m \times p}$ leads to the linear system specified by $(A+B K C, B, C)$, the transfer function of which shall be denoted by $\mathbf{G}^{K}$. A straightforward calculation shows that

$\mathbf{G}^{K}(s)=C(s I-A-B K C)^{-1} B=\mathbf{G}(s)(I-K \mathbf{G}(s))^{-1}$.

As usual, $H^{\infty}\left(\mathbb{C}^{p \times m}\right)$ denotes the space of holomorphic, bounded functions $\mathbb{C}_{0} \rightarrow \mathbb{C}^{p \times m}$, which is a Banach space when equipped with the norm

$$
\|\mathbf{H}\|_{H^{\infty}}:=\operatorname{essip}_{\omega \in \mathbb{R}}\|\mathbf{H}(i \omega)\| \quad \forall \mathbf{H} \in H^{\infty}\left(\mathbb{C}^{p \times m}\right) .
$$

For $\mathbb{F}=\mathbb{R}$ or $\mathbb{F}=\mathbb{C}$, let $\mathbb{S}_{\mathbb{F}}(\mathbf{G})$ denote the set of stabilising output feedback gains in $\mathbb{F}^{m \times p}$, that is,

$$
\mathbb{S}_{\mathbb{F}}(\mathbf{G}):=\left\{K \in \mathbb{F}^{m \times p}: \mathbf{G}^{K} \in H^{\infty}\left(\mathbb{C}^{p \times m}\right)\right\} .
$$

We shall typically impose the assumption that $(A, B, C)$ is stabilisable and detectable, in which case,

$$
\mathbb{S}_{\mathbb{F}}(\mathbf{G}):=\left\{K \in \mathbb{F}^{m \times p}: A+B K C \text { is Hurwitz }\right\} .
$$

The following result provides a characterisation of balls of stabilising complex feedback gains in terms of a related $H^{\infty}$-norm condition. A proof may be found in [10, Proposition 5.6] or [29, Lemma 2.1].

Lemma 2.2. For $K \in \mathbb{R}^{m \times p}$ and $r>0, \mathbb{B}_{\mathbb{C}}(K, r) \subseteq$ $\mathbb{S}_{\mathbb{C}}(\mathbf{G})$ if, and only if, $\left\|\mathbf{G}^{K}\right\|_{H^{\infty}} \leq 1 / r$.

We emphasize that Lemma 2.2 does not hold if in the statement $\mathbb{C}$ is replaced by $\mathbb{R}$. More specifically, the inclusion $\mathbb{B}_{\mathbb{R}}(K, r) \subseteq \mathbb{S}_{\mathbb{R}}(\mathbf{G})$ does in general not imply that $\left\|\mathbf{G}^{K}\right\|_{H^{\infty}} \leq 1 / r$.

We shall make extensive use of the following stability result, the so-called complex Aizerman conjecture, for the unforced system (1.1), see [16, Theorem 3.14 and Corollary 3.15] or [17, Theorem 5.6.22]. We note that the complex Aizerman conjecture is true, whereas it is well-known that the real Aizerman conjecture is false in general, see, for example, [15, Example 6.9] and [43].

Theorem 2.3. Given $\Sigma$ as in (2.1) and locally Lipschitz $f: \mathbb{R}^{p} \rightarrow \mathbb{R}^{m}$, assume that $(A, B, C)$ is stabilisable and detectable. Assume further that $K \in \mathbb{R}^{m \times p}$ and $r>0$ are such that $\mathbb{B}_{\mathbb{C}}(K, r) \subseteq \mathbb{S}_{\mathbb{C}}(\mathbf{G})$. If

$$
\|f(z)-K z\|<r\|z\| \quad \forall z \in \mathbb{R}^{p}, z \neq 0,
$$

then (1.1) is 0-GAS.

\section{Strong iISS for forced Lur'e systems}

The following theorem is the main result of the paper.

Theorem 3.1. Given $\Sigma$ as in (2.1) and locally Lipschitz $f: \mathbb{R}^{p} \rightarrow \mathbb{R}^{m}$, assume that $(A, B, C)$ is stabilisable and detectable. Assume further that $K \in \mathbb{R}^{m \times p}$ and $r>0$ are such that $\mathbb{B}_{\mathbb{C}}(K, r) \subseteq \mathbb{S}_{\mathbb{C}}(\mathbf{G})$, and that there exists $\alpha: \mathbb{R}_{+} \rightarrow \mathbb{R}_{+}$such that

$$
\|f(z)-K z\| \leq r\|z\|-\alpha(\|z\|) \quad \forall z \in \mathbb{R}^{p} .
$$

(1) If $\alpha \in \mathcal{K}$, then (1.1) is strongly iISS. Moreover, the iISS estimate (2.4) holds with

$$
\gamma_{2}(s)=a s+b s^{2} \quad \forall s \in \mathbb{R}_{+}
$$

for some $a>0$ and $b \geq 0$, with $b=0$ if $D_{\mathrm{e}}=0$.

(2) If $\alpha \in \mathcal{K}_{\infty}$, then (1.1) is ISS.

We provide some commentary on the above theorem. 
Remark 3.2. (i) Statement (1) is the main novel contribution of the present work. Statement (2) is a generalisation of [29, Theorem 3.1] which considers (1.1) with $D_{\mathrm{e}}=0$, and is included here for completeness. iISS and strong iISS are not considered in [29].

(ii) The claim that the estimate (2.4) holds with $\gamma_{2}$ of the form (3.2) implies that, for all $x^{0} \in \mathbb{R}^{n}$, the solution $x\left(\cdot ; x^{0}, v\right)$ of $(1.1)$ converges to zero if $v \in$ $L^{1}\left(\mathbb{R}_{+}, \mathbb{R}^{q}\right) \cap L^{2}\left(\mathbb{R}_{+}, \mathbb{R}^{q}\right)\left(v \in L^{1}\left(\mathbb{R}_{+}, \mathbb{R}^{q}\right)\right.$ in the case wherein $\left.D_{\mathrm{e}}=0\right)$, see Remark 2.1.

(iii) In Theorem 5.1, we present a condition in terms of the data $r, K$ and $f$, which is equivalent to the existence of a (evidently non-unique) function $\alpha \in \mathcal{K}$ (or $\alpha \in \mathcal{K}_{\infty}$ ) satisfying (3.1).

Invoking Lemma 2.2, the following nonlinear smallgain version of statement (1) of Theorem 3.1 is immediate.

Corollary 3.3. Given $\Sigma$ as in (2.1) and locally Lipschitz $f: \mathbb{R}^{p} \rightarrow \mathbb{R}^{m}$, assume that $(A, B, C)$ is stabilisable and detectable, and that $K \in \mathbb{S}_{\mathbb{R}}(\mathbf{G})$. If there exists $\alpha \in \mathcal{K}$ such that, for all $z \in \mathbb{R}^{p} \backslash\{0\}$,

$$
\left\|\mathbf{G}^{K}\right\|_{H^{\infty}} \frac{\|f(z)-K z\|}{\|z\|} \leq 1-\frac{\alpha(\|z\|)}{\|z\|},
$$

then (1.1) is strongly iISS. Moreover, the iISS estimate (2.4) holds with $\gamma_{2}$ of the form (3.2).

The following corollary of Theorem 3.1 shows that if $f$ is globally Lipschitz, then the function $\gamma_{2}$ in (2.4) can be chosen to be linear.

Corollary 3.4. Imposing the notation and assumptions of statement (1) of Theorem 3.1, if $f$ is globally Lipschitz, then the iISS estimate (2.4) holds with $\gamma_{2}(s)=$ as for some $a>0$.

In the context of small-signal ISS, it is important and interesting to compute or estimate the largest possible value for the input threshold $R$ appearing in (2.5) in terms of the data of the Lur'e system, including the comparison function $\alpha$ in (3.1) and (3.3). Whilst this is difficult in general (although see [6, Theorems 1 and 2]), we give a class of examples where the "optimal" value for $R$ turns out to be equal to $\lim _{s \rightarrow \infty} \alpha(s)$.

Example 3.5. The forced scalar differential equation

$$
\dot{x}=-x+f(x)+v, \quad x(0)=x^{0},
$$

where $f: \mathbb{R} \rightarrow \mathbb{R}$, is a special case of (1.1) with $A=$ -1 and $B=C=1=B_{\mathrm{e}}$ and $D_{\mathrm{e}}=0$. Here $\mathbf{G}(s)=$ $1 /(s+1)$ and so, trivially, $\|\mathbf{G}\|_{H^{\infty}}=\mathbf{G}(0)=1$. Hence, $\mathbb{B}_{\mathbb{C}}(K, r) \subseteq \mathbb{S}_{\mathbb{C}}(\mathbf{G})$ with $K=0$ and $r=1$. We shall revisit this example throughout the paper in various contexts.

Let $\phi \in \mathcal{K} \backslash \mathcal{K}_{\infty}$ be such that $\phi(s)<s$ for all $s>0$ and fix $f(x)=x-\operatorname{sign}(x) \phi(|x|)$ for all $x \in \mathbb{R}$. Then

$$
|f(x)|=|x|-\phi(|x|), \quad \forall x \in \mathbb{R},
$$

and so, choosing $\alpha=\phi$, condition (3.1) and the smallgain inequality (3.3) are satisfied. By Theorem 3.1 (or Corollary 3.3), the zero equilibrium pair is strongly iISS, and so, in particular, has the small signal ISS property. The closed-loop feedback system may be written as

$$
\dot{x}=-\operatorname{sign}(x) \phi(|x|)+v,
$$

and, setting $R^{*}:=\lim _{s \rightarrow \infty} \alpha(s)=\lim _{s \rightarrow \infty} \phi(s)$, it is clear that inputs $v$ with $\|v\|_{L^{\infty}}<R^{*}$ lead to bounded state trajectories. Furthermore, we claim that, for every $R \in\left(0, R^{*}\right)$, there exist $\beta \in \mathcal{K} \mathcal{L}$ and $\gamma \in \mathcal{K}$ such that (2.5) holds. To prove this, it is sufficient to show that, for any $R \in\left(0, R^{*}\right)$, there exist $\psi$ and $\lambda$ in $\mathcal{K}_{\infty}$ such that, for every solution $x$ generated by an input $v$ with $\|v\|_{L^{\infty}} \leq R$, we have

$$
\frac{1}{2} \frac{d}{d t} x^{2}(t) \leq-\psi(|x(t)|)+\lambda\left(\|v\|_{L^{\infty}}\right) \quad \text { a.e. } t \geq 0 \text {. }
$$

Choose $L>0$ such that $\phi(s)>R$ for all $s \geq L$, and set $\psi_{1}(s):=s(\phi(s)-R)$ for $s \geq L$. Let $\psi_{0}:[0, L] \rightarrow \mathbb{R}_{+}$ be strictly increasing such that $\psi_{0}(L)=\psi_{1}(L)$ and $\psi_{0}(s) \leq s \phi(s)$ and define a $\mathcal{K}_{\infty}$-function $\psi_{2}$ by

$$
\psi_{2}(s):= \begin{cases}\psi_{0}(s), & s \in[0, L) \\ \psi_{1}(s), & s \geq L .\end{cases}
$$

Then, for all for $v$ such that $\|v\|_{L^{\infty}} \leq R$,

$$
\begin{aligned}
\frac{1}{2} \frac{d}{d t} x^{2}(t) & =x(t) \dot{x}(t) \leq-|x(t)| \phi(|x(t)|)+|x(t)||v(t)| \\
& \leq-\psi_{2}(|x(t)|)+L|v(t)| \quad \text { a.e. } t \geq 0 .
\end{aligned}
$$

Consequently, with the choices $\psi=\psi_{2}$ and $\lambda(s)=L s$, inequality (3.5) holds for all inputs $v$ with $\|v\|_{L^{\infty}} \leq R$. Finally, we claim that the constant input $v(t) \equiv R^{*}$ generates divergent state trajectories: indeed, defining the function $F$ by

$$
F(z):=\int_{0}^{z} \frac{d \xi}{R^{*}-\operatorname{sign}(\xi) \phi(|\xi|)} \quad \forall z \in \mathbb{R},
$$

and invoking separation of variables, we see that the solution $x$ with initial value equal to 0 satisfies $F(x(t))=t$ for all $t \geq 0$, implying that $x(t) \rightarrow \infty$ as $t \rightarrow \infty$.

To conclude the example, we note that any complex feedback gain $k$ with $\operatorname{Re} k \geq 1=r$ destabilizes $\mathbf{G}$ and that, with our choice $\alpha=\phi$, conditions (3.1) and (3.3) hold with equality. This means that both $r$ and $\alpha$ are as large as they can be (for fixed $K=0$ ), and thus, in this sense, the scenario considered in the example is "extreme".

We note that although the model data $\Sigma$ and $f$ in Theorem 3.1 are assumed to be real, a key hypothesis is that every feedback gain in the complex ball $\mathbb{B}_{\mathbb{C}}(K, r)$ is stabilising for $\mathbf{G}$. Our next result shows that the complex ball condition may be weakened to a real ball condition, provided that a suitable additional assumption is satisfied. We say that a proper rational 
matrix $\mathbf{H} \in H^{\infty}\left(\mathbb{C}^{p \times m}\right)$ has the real supremum value property if there exists $s_{*} \in\{s \in \mathbb{C}: \operatorname{Re} s \geq 0\} \cup\{\infty\}$ such that

$$
\|\mathbf{H}\|_{H^{\infty}}=\left\|\mathbf{H}\left(s_{*}\right)\right\| \quad \text { and } \quad \mathbf{H}\left(s_{*}\right) \in \mathbb{R}^{p \times m},
$$

where $\mathbf{H}(\infty):=\lim _{|s| \rightarrow \infty} \mathbf{H}(s)$. We are now in position to state the following corollary to Theorem 3.1 .

Corollary 3.6. Given $\Sigma$ as in (2.1) and locally Lipschitz $f: \mathbb{R}^{p} \rightarrow \mathbb{R}^{m}$, assume that $(A, B, C)$ is stabilisable and detectable. Assume further that $K \in \mathbb{R}^{m \times p}$ and $r>0$ are such that $\mathbb{B}_{\mathbb{R}}(K, r) \subseteq \mathbb{S}_{\mathbb{R}}(\mathbf{G})$ and $\mathbf{G}^{K}$ has the real supremum value property. Then statements (1) and (2) of Theorem 3.1 hold.

We provide some classes of systems for which the real supremum value assumption is satisfied.

Example 3.7. The following examples have the property that

$$
\left\|\mathbf{G}^{K}\right\|_{H^{\infty}}=\left\|\mathbf{G}^{K}(0)\right\| .
$$

Since $\mathbf{G}^{K}(0)$ is real, it follows that $\mathbf{G}^{K}$ satisfies the real supremum value assumption.

(i) Recall that a square matrix is called Metzler if every off-diagonal entry is nonnegative (see, for example, [4, Ch. 6]). If $(A, B, C)$ and $K$ are such that $A+B K C$ is Hurwitz and Metzler and $B$ and $C$ are nonnegative, that is $B \in \mathbb{R}_{+}^{n \times m}$ and $C \in \mathbb{R}_{+}^{p \times n}$, then $\mathbf{G}^{K}$ is the transfer function of a stable positive system, and so satisfies (3.6) by, for instance, [18, Theorem 5].

(ii) If $K$ and $(A, B, C)$ are such that $(A+B K C, B, C)$ is a so-called symmetric system, meaning $A+B K C=$ $(A+B K C)^{T}$ and $C=B^{T}$, then (3.6) holds, see $[25$, Remark 4.1 2.].

(iii) Let $\left(\tilde{A}, \tilde{b}, \tilde{c}^{T}\right) \in \mathbb{R}^{n \times n} \times \mathbb{R}^{n} \times \mathbb{R}^{1 \times n}$ with Hurwitz $\tilde{A}$, transfer function $\mathbf{H}$, and let $g$ be a real parameter. Consider the integral control feedback system

$$
\dot{x}=\tilde{A} x+\tilde{b} u, \quad y=\tilde{c}^{T} x, \quad \dot{u}=w-g y,
$$

where $w$ is an external input. This $(n+1)$-dimensional system (with input $w$ and output $y$ ) is described by the triple $\left(A_{g}, b, c^{T}\right)$, where

$$
A_{g}:=\left(\begin{array}{cc}
\tilde{A} & \tilde{b} \\
-g \tilde{c}^{T} & 0
\end{array}\right), \quad b:=\left(\begin{array}{l}
0 \\
1
\end{array}\right), \quad c^{T}:=\left(\begin{array}{cc}
\tilde{c}^{T} & 0
\end{array}\right) .
$$

The transfer function $\mathbf{G}_{g}$ of the triple $\left(A_{g}, b, c^{T}\right)$ is given by

$$
\mathbf{G}_{g}(s)=\frac{\mathbf{H}(s)}{s+g \mathbf{H}(s)} .
$$

It follows from [27, Proposition 3.9] that if $\mathbf{H}(0) \neq$ 0 , then there exists $g^{*}>0$ such that, for all $g$ with $g \mathbf{H}(0)>0$ and $0<|g|<g^{*}$,

$$
\left\|\mathbf{G}_{g}\right\|_{H^{\infty}}=\frac{1}{|g|}=\left|\mathbf{G}_{g}(0)\right| \text {. }
$$

In particular, (3.6) holds with $\mathbf{G}=\mathbf{G}_{g}$ and $K=0 . \diamond$
Next we present a version of Theorem 3.1 which is reminiscent of the well-known circle criterion [14, 22, 41]. To this end, recall that a square proper rational matrix-valued function $s \mapsto \mathbf{H}(s)$ of a complex variable $s$ is said to be positive real if for every $s \in \mathbb{C}_{0}$, which is not a pole of $\mathbf{H}$, the matrix $[\mathbf{H}(s)]^{*}+\mathbf{H}(s)$ is positive semi-definite. Here the superscript $*$ denotes the Hermitian transpose. It follows from [10, Proposition 3.3] that if $\mathbf{H}$ is positive real, then $\mathbf{H}$ is holomorphic on $\mathbb{C}_{0}$.

Corollary 3.8. Given $\Sigma$ as in (2.1) and locally Lipschitz $f: \mathbb{R}^{p} \rightarrow \mathbb{R}^{m}$, assume that $(A, B, C)$ is stabilisable and detectable. Assume further that $K_{1}, K_{2} \in$ $\mathbb{R}^{m \times p}$ are such that $\left(I-K_{2} \mathbf{G}\right)\left(I-K_{1} \mathbf{G}\right)^{-1}$ is positive real, and there exists $\alpha: \mathbb{R}_{+} \rightarrow \mathbb{R}_{+}$such that, for all $z \in \mathbb{R}^{p}$

$$
\left\langle f(z)-K_{1} z, f(z)-K_{2} z\right\rangle \leq-\|z\| \alpha(\|z\|) .
$$

(1) If $\alpha \in \mathcal{K}$, then (1.1) is strongly iISS. Moreover, the iISS estimate (2.4) holds with $\gamma_{2}$ of the form (3.2).

(2) If $\alpha \in \mathcal{K}_{\infty}$, then (1.1) is ISS.

The remark below provides some commentary on the above corollary.

Remark 3.9. (i) Statement (1) of Corollary 3.8 is the primary novel contribution of the present work in the context of the circle criterion - statement (2) is a generalisation of [29, Corollary 3.10] which considers (1.1) with $D_{\mathrm{e}}=0$, and is included for completeness.

(ii) Corollary 3.8 is closely related to the classical circle criterion, which is known to be sufficient for global asymptotic stability of unforced Lur'e system, see [13, Theorem 5.1], [14, Corollary 5.8] and [22, Theorem 7.1]. The term "circle criterion" is motivated by the graphical interpretation of the positive-real condition in the single-input single-output case $(m=p=1)$, see [22, pp. 266-270]. In the formulation of the classical circle criterion it is usually assumed that:

(a) $K_{1}, K_{2} \in \mathbb{R}^{m \times p}$ are such that $K_{1}-K_{2}$ is left invertible;

(b) $\mathbf{H}:=\left(I-K_{2} \mathbf{G}\right)\left(I-K_{1} \mathbf{G}\right)^{-1}$ is strictly positive real, meaning there exists $\varepsilon>0$ such that the function $s \mapsto \mathbf{H}(s-\varepsilon)$ is positive real;

(c) $f$ satisfies the sector condition $\left\langle f(z)-K_{1} z, f(z)-\right.$ $\left.K_{2} z\right\rangle \leq 0 \quad \forall z \in \mathbb{R}^{p}$.

It is know that (a)-(c) together imply that the hypotheses of Corollary 3.8 are satisfied with some $\alpha \in$ $\mathcal{K}_{\infty}$ (see the proofs of [29, corollaries 3.10 and 3.11$]$ ). Consequently, if (a)-(b) hold, then (1.1) is ISS, and, a fortiori, strongly iISS.

(iii) In the paper [2], an ISS result is obtained for the Lur'e system (1.1) under the assumptions that $B_{\mathrm{e}}=B$ and $D_{\mathrm{e}}=0$, the underlying linear system is positive real, and the nonlinearity (which may have superlinear growth) satisfies a suitable cone condition, see [2, 
Theorem 1]. The overlap between Corollary 3.8 and [2, Theorem 1] is very small as [2] focusses solely on ISS and differs in the assumptions on the linear system. $\diamond$

Our final result of this section demonstrates that, under an additional assumption on the linear system, a well-known condition which is sufficient for 0-GAS of the unforced system (1.1) (see Theorem 3.1) guarantees ilSS of (1.1).

Proposition 3.10. Given $\Sigma$ as in (2.1) and locally Lipschitz $f: \mathbb{R}^{p} \rightarrow \mathbb{R}^{m}$, assume that $(A, B, C)$ is (i) stabilisable and observable, or (ii) controllable and detectable. Assume further that $K \in \mathbb{R}^{m \times p}$ and $r>0$ are such that $\mathbb{B}_{\mathbb{C}}(K, r) \subseteq \mathbb{S}_{\mathbb{C}}(\mathbf{G})$ and that (2.6) is satisfied. Then (1.1) is iISS and the iISS estimate (2.4) holds with $\gamma_{2}$ of the form (3.2).

By way of comparing the conditions (3.1) and (2.6), note that the latter is equivalent to the existence of a continuous function $\alpha: \mathbb{R}_{+} \rightarrow \mathbb{R}_{+}$such that $\alpha(0)=0$, $\alpha(s)>0$ for $s>0$ and

$$
\|f(z)-K z\| \leq r\|z\|-\alpha(\|z\|) \quad \forall z \in \mathbb{R}^{p} .
$$

We do not know whether or not the controllability/observability assumption in Proposition 3.10 is necessary. What is clear, as the following example demonstrates, is that the condition (2.6), which, by Proposition 3.10, is sufficient for iISS, is not sufficient for strong iISS.

Example 3.11. Consider the scalar Lur'e system (3.4) from Example 3.5. The triple $(A, B, C)=(-1,1,1)$ is trivially controllable and observable. Let $f$ in (3.4) be given by

$$
f: \mathbb{R} \rightarrow \mathbb{R}, \quad f(z)=z-z e^{-z^{2}} \quad \forall z \in \mathbb{R} .
$$

Obviously, with the choices $K=0$ and $r=1$, there does not exist $\alpha \in \mathcal{K}$ such that (3.1) is satisfied, but (2.6) does hold. In particular, by Proposition 3.10, the zero equilibrium pair is iISS.

However, for each $\varepsilon>0$, it is possible to choose $x^{0}$ sufficiently large so that $x\left(t ; x^{0}, \varepsilon\right)$ (with constant input $\varepsilon)$ diverges as $t \rightarrow \infty$. We conclude that the smallsignal ISS property does not hold, and so the zero equilibrium pair is not strongly iISS.

$\diamond$

Remark 3.12. Consider the situation wherein the nonlinear term $f$ in (1.1) is assumed to be time-varying, that is, the differential equation in (1.1) is replaced by

$$
\dot{x}(t)=A x(t)+B f\left(t, C x(t)+D_{\mathrm{e}} v(t)\right)+B_{\mathrm{e}} v(t),
$$

where $f$ has enough regularity to ensure that, for all $x^{0} \in \mathbb{R}^{n}$ and $v \in L_{\text {loc }}^{\infty}\left(\mathbb{R}_{+}, \mathbb{R}^{q}\right)$, a unique absolutely continuous solution $x\left(\cdot ; x^{0}, v\right)$ of $(3.8)$ exists. It is not difficult to see that the conclusions of Theorem 3.1 and Proposition 3.10 continue to hold if the conditions (3.1) and (2.6) are satisfied uniformly in time, that is,

$\|f(t, z)-K z\| \leq r\|z\|-\alpha(\|z\|) \quad \forall(t, z) \in \mathbb{R}_{+} \times \mathbb{R}^{p}$, and

$$
\sup _{t \geq 0}\|f(t, z)-K z\|<r\|z\| \quad \forall z \in \mathbb{R}^{p}, z \neq 0
$$

respectively. The same is true of Corollary 3.8, provided that the sector condition (3.7) holds uniformly in time.

\section{Strong iISS for Lur'e systems with saturating nonlinearities}

The present section is motivated by [29, Proposition 3.4] and the recent paper [3]. The former result states that if, in the Lur'e system (1.1) the matrix $A$ is not Hurwitz and $f$ is bounded, then the $(0,0)$ equilibrium cannot be ISS. The situation wherein $A$ is not Hurwitz and $f$ is bounded arises, for instance, in the stabilisation of linear systems $(2.2 \mathrm{a})$ by saturated feedback. The main result in this section provides sufficient conditions for strong iISS of forced Lur'e systems for a given class of nonlinearities which includes saturation functions, and strengthens the result [3, Theorem 2].

To be specific, we consider the class $\mathcal{F}$ of locally Lipschitz functions $f: \mathbb{R}^{m} \rightarrow \mathbb{R}^{m}$ which have the following two properties:

$(\mathcal{F} .1)\|f(w)\|^{2} \leq\langle f(w), w\rangle$ for $w \in \mathbb{R}^{m} ;$

$(\mathcal{F} .2)$ there exists $\beta, \gamma, \delta>0$ such that, for all $w \in \mathbb{R}^{m}$

$$
\begin{array}{ll}
\langle f(w), w\rangle \geq \beta\|w\|^{2} & \text { if }\|w\| \leq \delta \\
\text { and } & \langle f(w), w\rangle \geq \gamma\|w\| \quad \text { if }\|w\| \geq \delta .
\end{array}
$$

The first condition $(\mathcal{F} .1)$ states that $f$ belongs to the sector $[0, I]$ in the terminology of $[22$, Definition 6.2 , p. 232] and, if it holds, then evidently $f(0)=0$. Property $(\mathcal{F} .2)$ is the same as that imposed in $[7$, Theorem $2.2]$.

Before stating our main result of the section we provide two families of saturation functions which belong to $\mathcal{F}$, the first of which is not "diagonal". The proofs that these functions belong to $\mathcal{F}$ are elementary exercises, and are therefore omitted.

Example 4.1. (i) For all $\delta>0$, the function $\theta: \mathbb{R}^{m} \rightarrow$ $\mathbb{R}^{m}$ defined by $\theta(w)=w$ if $\|w\| \leq \delta$ and $\theta(w)=$ $\delta w /\|w\|$ if $\|w\| \geq \delta$ belongs to $\mathcal{F}$. The function $\theta$ is, up to a sign change, equal to the saturation function considered in [33], see [33, equation (2.8)].

(ii) The ubiquitous diagonal saturation function, see for example [22, p. 19], always belongs to $\mathcal{F}$.

Theorem 4.2. Given $\Sigma$ as in (2.1), assume that $(A, B, C)$ is stabilisable and detectable. If there exists $K \in \mathbb{R}^{m \times p}, K \neq 0$, such that $\mathbb{B}_{\mathbb{C}}(K,\|K\|) \subseteq \mathbb{S}_{\mathbb{C}}(\mathbf{G})$, then, for every $g \in \mathcal{F}$ the forced Lur'e system

$$
\dot{x}=A x+B g\left(K C x+K D_{\mathrm{e}} v\right)+B_{\mathrm{e}} v, \quad x(0)=x^{0},
$$

is strongly iISS, and the iISS estimate (2.4) holds with $\gamma_{2}$ of the form (3.2). 
We give two examples of classes of systems where the ball condition $\mathbb{B}_{\mathbb{C}}(K,\|K\|) \subseteq \mathbb{S}_{\mathbb{C}}(\mathbf{G})$ is satisfied.

Example 4.3. (i) If $\mathbf{G}$ is positive real, then for every $k>0$, it follows from [10, Lemma 2.4] and [10, Theorem 6.4$]$ that $\mathbb{B}_{\mathbb{C}}(-k I, k) \subseteq \mathbb{S}_{\mathbb{C}}(\mathbf{G})$, and so the ball condition in Theorem 4.2 holds with $K=-k I$.

(ii) Suppose that $m=p$ and the transfer function $\mathbf{G}$ of $(A, B, C)$ has the form $\mathbf{G}(s)=\mathbf{H}(s) / s$, for $\mathbf{H} \in H^{\infty}\left(\mathbb{C}^{m \times m}\right)$ with $\mathbf{H}(0)=\mathbf{H}(0)^{*}$ positive definite. Then, by [27, Proposition 3.9], there exists $k^{*}>0$ such that $\mathbf{G}^{k}=\mathbf{G}(I-k \mathbf{G})^{-1} \in H^{\infty}$ and $\left\|\mathbf{G}^{k}\right\|_{H^{\infty}}=1 /|k|$ for all $k \in\left(-k^{*}, 0\right)$. Consequently, by Lemma 2.2 , the ball condition in Theorem 4.2 is satisfied with $K=k I$ for all $k \in\left(-k^{*}, 0\right)$.

Theorem 4.2 generalises [3, Theorem 2] by imposing weaker assumptions than [3, Theorem 2]. To see this, we recall the definitions of the sets of saturation functions $\mathcal{S}$ and $\mathcal{S}^{m}$ considered in [3], see also [24]. A function $\sigma: \mathbb{R} \rightarrow \mathbb{R}$ belongs to $\mathcal{S}$ if $\sigma$ is locally Lipschitz, bounded, $s \sigma(s)>0$ for all nonzero $s$, $\liminf _{s \rightarrow 0} \sigma(s) / s>0$ and $\liminf _{|s| \rightarrow \infty} \sigma(s)>0$. For $m \in \mathbb{N}$, the set $\mathcal{S}^{m}$ comprises functions $\sigma: \mathbb{R}^{m} \rightarrow \mathbb{R}^{m}$ such that $(\sigma(z))_{i}=\sigma_{i}\left(z_{i}\right)$ for some $\sigma_{i} \in \mathcal{S}$, for all $i \in\{1, \ldots, m\}$ and all $z=\left(z_{1}, z_{2}, \ldots, z_{m}\right)^{T} \in \mathbb{R}^{m}$. The following proposition relates the class of functions $\mathcal{S}^{m}$ to $\mathcal{F}$.

Proposition 4.4. For every $\sigma \in \mathcal{S}^{m}$, there exists $l>$ 0 such that the function $z \mapsto \sigma(l z)$ is in $\mathcal{F}$.

We comment that we should not, in general, expect a converse of Proposition 4.4 to hold as $\mathcal{F}$ contains functions which are not "diagonal".

Remark 4.5. (i) To see that [3, Theorem 2] follows from Theorem 4.2, we note that [3] considers the forced Lur'e system (1.1) with linear component $\left(A,-B, B^{*}\right)$ where $A=-A^{*}$, and nonlinearity $\sigma \in$ $\mathcal{S}^{m}$. Since the triple $\left(A, B, B^{*}\right)$ is positive real by, for example, [10, Corollary 7.4], an application of part (1) of Example 4.3 yields that $\mathbb{B}_{\mathbb{C}}(k I, k) \subseteq \mathbb{S}_{\mathbb{C}}(\mathbf{G})$ for all $k>0$. Moreover, [3, Theorem 2] assumes that $(A, B)$ is controllable, so that $\left(B^{*},-A^{*}\right)$ is observable, and thus $\left(A,-B, B^{*}\right)$ is stabilisable and detectable. By Proposition 4.4, there exists $l>0$ such that the function $z \mapsto \sigma(l z)$ is in $\mathcal{F}$. Therefore, strong iISS of the zero equilibrium pair follows from Theorem 4.2 with $k:=1 / l>0$, as evidently $\sigma(s)=\sigma(l k s)$ for all $s \in \mathbb{R}$. Consequently, the skew symmetric structure and diagonal saturation functions imposed in [3, Theorem 2] are not required.

(ii) We provide some comments on related literature. To this end, consider the controlled linear system with nonlinear feedback

$$
\dot{x}=A x+\Phi(x), \quad x(0)=x^{0},
$$

with $\Phi$ a bounded function. The papers [38, 39] investigate asymptotic stability of (4.2) and [23] considers semi-global exponential stabilisation of (4.2) by (diagonally) saturated linear feedback. These three papers do not consider ISS-type stability notions - no external forcing is present. The paper [24] primarily considers (input/output) $L^{p}$-stability of (1.1) (for all $p \in[1, \infty])$ with $x(0)=0, f=\sigma \in \mathcal{S}^{m}$, and a small bounded forcing term $B_{\mathrm{e}} v$ (in the notation of the current paper). Whilst [24] contains a result of ISS-type (see $[24$, inequality $(60)]$ ), it does not provide conditions which guarantee the iISS property. We note that the relevant results in $[23,24]$ are different to Theorem 4.2: in $[23,24]$, for a fixed saturation function, the existence of a linear stabilising feedback is established. In contrast, we assume that $K$ is a stabilising feedback for the linear system specified by $(A, B, C)$. Theorem 4.2 says that if $K$ additionally satisfies the ball condition $\mathbb{B}_{\mathbb{C}}(K,\|K\|) \subseteq \mathbb{S}_{\mathbb{C}}(\mathbf{G})$, then the forced Lur'e system (4.1) is strongly iISS for all saturation functions $g \in \mathcal{F}$.

\section{Discussion}

We conclude by placing the findings of this paper into the context given by related results in the literature, thereby providing a wider perspective. As usual, we assume that the nonlinearity $f$ in (1.1) satisfies $f(0)=$ 0 . Recall that the unforced Lur'e system (1.1) is said to be stable in the large if there exists $M>0$ such that, for every initial condition $x^{0}$,

$$
\left\|x\left(t ; x^{0}, 0\right)\right\| \leq M\left\|x^{0}\right\| \quad \forall t \geq 0 .
$$

Furthermore, (1.1) is said to be exponentially ISS if there exist $M, \gamma>0$ such that, for all $v \in$ $L_{\text {loc }}^{\infty}\left(\mathbb{R}_{+}, \mathbb{R}^{q}\right)$, all $x^{0} \in \mathbb{R}^{n}$, and all $t \geq 0$

$$
\left\|x\left(t ; x^{0}, v\right)\right\| \leq M\left(e^{-\gamma t}\left\|x^{0}\right\|+\|v\|_{L^{\infty}(0, t)}\right) .
$$

Note that exponential ISS of (1.1) implies that the zero equilibrium of the associated unforced system is globally exponentially stable. Finally, we say that (1.1) is semi-globally exponentially ISS if, for all $\Gamma>0$, there exist $M, \gamma>0$ such that (5.1) holds for all $v \in L^{\infty}\left(\mathbb{R}_{+}, \mathbb{R}^{q}\right)$ and $x^{0} \in \mathbb{R}^{n}$ such that

$$
\left\|x^{0}\right\|+\|v\|_{L^{\infty}} \leq \Gamma .
$$

For all practical purposes the above semi-global exponential ISS property is adequate: for any given application context, there exists a $\Gamma>0$ such that every practically relevant initial condition $x^{0}$ and forcing term $v$ combined will satisfy (5.2).

The following theorem provides an overview of stability results for (1.1) and its unforced version, formulated in the spirit of the complex Aizerman conjecture.

Theorem 5.1. Given $\Sigma$ as in (2.1) and locally Lipschitz $f: \mathbb{R}^{p} \rightarrow \mathbb{R}^{m}$, assume that $(A, B, C)$ is stabilisable and detectable, and let $K \in \mathbb{R}^{m \times p}, r>0$ be such that $\mathbb{B}_{\mathbb{C}}(K, r) \subseteq \mathbb{S}_{\mathbb{C}}(\mathbf{G})$. Define $\Delta: \mathbb{R}^{p} \rightarrow \mathbb{R}$ by

$$
\Delta(z):=r\|z\|-\|f(z)-K z\| \quad \forall z \in \mathbb{R}^{p} .
$$

The following statement holds.

(1) If $\Delta(z) \geq 0$ for all $z \in \mathbb{R}^{p}$, then (1.1) is stable in the large. 
Furthermore, assuming that $\Delta(z)>0$ for all $z \in$ $\mathbb{R}^{p} \backslash\{0\}$, the following statements hold.

(2) System (1.1) is 0-GAS. If additionally $(A, B, C)$ is controllable or observable, then (1.1) is iISS.

(3) If $\liminf _{\|z\| \rightarrow \infty} \Delta(z)>0$, then (1.1) is strongly iISS.

(4) If $\Delta$ is radially unbounded, then (1.1) is ISS.

(5) If $\Delta$ is radially unbounded and $\liminf _{\|z\| \rightarrow 0} \frac{\Delta(z)}{\|z\|}>0$, then (1.1) is semi-globally exponentially ISS.

(6) If $\liminf _{\|z\| \rightarrow 0} \frac{\Delta(z)}{\|z\|}>0$ and $\liminf _{\|z\| \rightarrow \infty} \frac{\Delta(z)}{\|z\|}>0$, then (1.1) is exponentially ISS.

Observe that $\Delta(z)$ is an indicator of how close $f(z)$ is to the boundary of the ball centred at $K z$ of radius $r\|z\|$. A key assumption in Theorem 5.1 is that every linear feedback in the ball $\mathbb{B}_{\mathbb{C}}(K, r)$ is stabilising. The conditions (1)-(6) require that $f$ is "further from the boundary" of the ball in increasingly strong senses with particular emphasis of the behaviour of $f$ near the origin and at infinity.

The following example demonstrates that the hypotheses in (1)-(5) in Theorem 5.1 do not, in general, imply the stability property guaranteed by that in the subsequent hypothesis.

Example 5.2. We consider again the scalar Lur'e system (3.4) from Example 3.5. As described there, (3.4) satisfies $\mathbb{B}_{\mathbb{C}}(K, r) \subseteq \mathbb{S}_{\mathbb{C}}(\mathbf{G})$ with $K=0$ and $r=1$, where $\mathbf{G}(s)=1 /(1+s)$. Trivially, in the context of the scalar Lur'e system (3.4), the triple $(A, B, C)$ is controllable and observable.

(i) For $f$ given by $f(z)=z$, we have that $\Delta(z) \equiv 0$, and so, by statement (1), the uncontrolled system (3.4) is stable in the large. Obviously, the condition $\Delta(z)>$ 0 is violated for every $z \neq 0$. Trivially, in this example, the solution of of the uncontrolled system (3.4) is constant, $x(t) \equiv x^{0}$, and so the zero equilibrium is not globally asymptotically stable.

(ii) Example 3.11 shows that the condition $\Delta(z)>0$ for all $z \in \mathbb{R}^{p} \backslash\{0\}$ is not sufficient for strong iISS of the forced Lur'e system (3.4).

(iii) It is straightforward to find nonlinearities for which $\Delta$ is bounded, $\Delta(z)>0$ for all $z \in \mathbb{R}^{p} \backslash\{0\}$ and $\liminf _{\|z\| \rightarrow \infty} \Delta(z)>0$ (so that, by statement (3), the system (3.4) is strongly iISS), but (3.4) fails to be ISS, see [29].

(iv) Fixing $\kappa \in(0,1)$, consider (3.4) with $f: \mathbb{R} \rightarrow \mathbb{R}$ given by

$$
f(z)= \begin{cases}z-z^{3} & |z| \in[0, \kappa) \\ z-\kappa^{3} \ln \left(\frac{|z|}{\kappa} e\right) & |z| \geq \kappa .\end{cases}
$$

Then $\Delta(z)>0$ for all $z \neq 0$ and $\Delta$ is radially unbounded, and thus, statement (4) guarantees that (3.4) is ISS. The lim inf assumption of statement (5) does not hold, however. Note that, for $x^{0} \in(0, \kappa)$ and $v(t) \equiv 0$, the solution of (3.4) is given by

$$
x(t)=\sqrt{\frac{\left(x^{0}\right)^{2}}{2 t\left(x^{0}\right)^{2}+1}} \quad \forall t \geq 0 .
$$

This function does not converge to zero exponentially, and consequently, (3.4) cannot be semi-globally exponentially ISS.

(v) Finally, consider (3.4) with $f: \mathbb{R} \rightarrow \mathbb{R}$ given by

$$
f(z)= \begin{cases}z-\frac{1}{2} z & |z| \in[0,1] \\ z-\frac{1}{2} \operatorname{sign}(z)|z|^{\frac{1}{2}} & |z|>1 .\end{cases}
$$

The function $f$ in (5.4) is such that $\Delta(z)>0$ for all $z \neq 0, \Delta$ is radially unbounded and $\Delta(z) / z$ satisfies the $\lim \inf$ condition at $z=0$, but not that at infinity. Hence, by statement (5), the system (3.4) with $f$ given by (5.3) is semi-globally exponentially ISS. However, for $x^{0}>1$ and $v(t) \equiv 0$, the solution $x$ satisfies

$$
x(t)=\left(x^{0}-\frac{1}{4} t\right)^{2} \quad \forall t \in\left[0,4\left(x^{0}-1\right)\right] .
$$

We claim that there does not exist $M, \gamma>0$ such that, for all $x^{0} \in \mathbb{R}$

$$
|x(t)| \leq M e^{-\gamma t}\left|x^{0}\right| \quad \forall t \geq 0 .
$$

Indeed, seeking a contradiction, if such $M, \gamma>0$ were to exist, then choose $x^{0}>1$ large enough so that

$$
t_{*}:=\frac{1}{\gamma} \log \left(M x^{0}\right)<4 x^{0}-4,
$$

and so $x^{0}-t_{*} / 4>1$. Evaluating $x$ at $t_{*}$, we obtain the contradiction

$$
1=M e^{-\gamma t_{*}} x^{0} \geq x\left(t_{*} ; x^{0}, 0\right)=\left(x^{0}-\frac{1}{4} t_{*}\right)^{2}>1 .
$$

We conclude that (3.4) with $v(t) \equiv 0$ is not exponentially stable, and, a fortiori, cannot be exponentially ISS.

\section{Summary}

To summarise, we have derived sufficient conditions for iISS and strong iISS of the zero equilibrium pair of finite-dimensional, continuous-time, forced Lur'e systems. Strong iISS is the combination of iISS and smallsignal ISS, and was introduced in [6]. Our main result, Theorem 3.1, says that if a complex ball $\mathbb{B}_{\mathbb{C}}(K, r)$ of linear static output-feedback gains is stabilising for a given linear system, then the zero equilibrium pair of the related forced Lur'e system (1.1) is strongly iISS for all nonlinearities $f$ for which there exists $\alpha \in \mathcal{K}$ such that $\|f(z)-K z\| \leq r\|z\|-\alpha(\|z\|)$ for all $z$. Under a weaker assumption on the nonlinearity, Proposition 3.10 provides a similar sufficient condition for 
iISS. We note that these results are reminiscent of the complex Aizerman conjecture (a well-known result in absolute stability theory $[16,17])$ and resonate with recent work on ISS of Lur'e systems [29]. Consequences of Theorem 3.1 include Corollary 3.8 and Theorem 4.2: the former extends the classical circle criterion to a strong iISS setting, the latter provides a sufficient condition for stabilisation of linear systems by saturated feedback and is related to the recent work [3].

\section{A Appendix}

\section{A.1 Proof of Theorem 3.1}

We collect some notation and preliminary technical results used in the proof. For positive semi-definite symmetric $M \in \mathbb{R}^{n \times n}$, we define the semi-norm $|\cdot|_{M}$ via

$$
|z|_{M}^{2}:=\langle z, M z\rangle \quad \forall z \in \mathbb{R}^{n} .
$$

We note that $|z|_{M}=0$ if, and only if, $z \in \operatorname{ker} M$. Moreover, $|z|_{M}=\left\|M^{1 / 2} z\right\|$, where $M^{1 / 2}$ denotes the unique positive semi-definite square root of $M$. Consequently, we obtain the Cauchy-Schwarz inequality $\left\langle z_{1}, M z_{2}\right\rangle \leq\left|z_{1}\right|_{M}\left|z_{2}\right|_{M}$ for all $z_{1}, z_{2} \in \mathbb{R}^{n}$ which we shall use extensively. Obviously, $|\cdot|_{M}$ is a norm if, and only if, $M$ is positive-definite. The operator seminorm induced by $|\cdot|_{M}$ is denoted by the same symbol. In particular, if $N \in \mathbb{R}^{n \times m}$, then $|N|_{M}=0$ if, and only if, $\operatorname{im} N \subset \operatorname{ker} M$. Finally, a straightforward calculation shows that $\nabla|z|_{M}=M z /|z|_{M}$ for all $z \in \mathbb{R}^{n}$ with $|z|_{M} \neq 0$, where $\nabla$ denotes the gradient.

The theorem below is a combination of $[1$, Theorem 1] and [1, Remark II.3], interpreted in the context of the Lur'e system (1.1).

Theorem A.1. If (1.1) is 0-GAS, and there exist a continuously differentiable radially unbounded function $V: \mathbb{R}^{n} \rightarrow \mathbb{R}_{+}$with $V(0)=0$ and $V(z)>0$ for $z \neq 0$, and $\zeta \in \mathcal{K}$, such that, for all $(z, w) \in \mathbb{R}^{n} \times \mathbb{R}^{q}$

$$
\left\langle(\nabla V)(z), A z+B f(y)+B_{\mathrm{e}} w\right\rangle \leq \zeta(\|w\|),
$$

where $y=C z+D_{\mathrm{e}} w$, then (1.1) is iISS.

Lemma A.2. Let $\kappa \in \mathcal{K}$ and $g_{1}>0$ be such that $2 g_{1}<\sup \kappa$ and set $g_{2}:=2 \kappa^{-1}\left(2 g_{1}\right)$. Then

$$
2 s w \leq s \kappa(s)+g_{2} w \quad \forall s \geq 0, \forall w \in\left[0, g_{1}\right] .
$$

For $\kappa \in \mathcal{K}_{\infty}$, the above lemma follows immediately from [21, Lemma 17], and the extension to $\kappa \in \mathcal{K}$ is straightforward. For completeness, we point out that if $\sup \kappa<\infty$ (that is, $\kappa \notin \mathcal{K}_{\infty}$ ) and if $g_{1}>0$ is such that $2 g_{1}>\sup \kappa$, then there does not exist a constant $g_{2}>0$ such that (A.2) holds.

Let $K \in \mathbb{R}^{m \times p}$ be as in Theorem 3.1 and set

$$
\left.\begin{array}{rl}
A^{K} & :=A+B K C, \quad B_{\mathrm{e}}^{K}:=B_{\mathrm{e}}+B K D_{\mathrm{e}} \\
\text { and } f^{K} & :=f-K .
\end{array}\right\}
$$

Lemma A.3. Imposing the notation and assumptions of Theorem 3.1, there exists positive semi-definite $P=$ $P^{T} \in \mathbb{R}^{n \times n}$ such that

$$
\left.\begin{array}{rl}
\left(A^{K}\right)^{T} P+P A^{K}+r^{2} C^{T} C & =-L^{T} L, \\
P B & =-L^{T},
\end{array}\right\}
$$

and a constant $d>0$ such that

$$
\|C z\| \leq d|z|_{P} \quad \forall z \in \mathbb{R}^{n} .
$$

Further, there exist positive-definite $Q=Q^{T} \in \mathbb{R}^{n \times n}$ and $\delta>0$ such that

$$
\begin{gathered}
\left\langle A^{K} z+B u, Q z\right\rangle+\left\langle z, Q\left(A^{K} z+B u\right)\right\rangle \\
\leq-\delta\|z\|^{2}+\|u\|^{2} \quad \forall z \in \mathbb{R}^{n}, \forall u \in \mathbb{R}^{m} .
\end{gathered}
$$

Without loss of generality, we may assume that $P \neq 0$, as if $P=0$, then $C=0$ by (A.5). Therefore, $A$ is Hurwitz by the detectability assumption. Moreover, the Lur'e system (1.1) reduces to

$$
\dot{x}=A x+B f\left(D_{\mathrm{e}} v\right)+B_{\mathrm{e}} v,
$$

which is easily seen to be exponentially ISS, and so is trivially iISS with linear $\gamma_{2}$ in the iISS estimate (2.4).

Proof of Lemma A.3. Since $\mathbf{G}^{K} \in H^{\infty}\left(\mathbb{C}^{p \times m}\right)$, it follows from the stabilisability and detectability assumptions that $A^{K}:=A+B K C$ is Hurwitz. Moreover, $\left\|\mathbf{G}^{K}\right\|_{H^{\infty}} \leq 1 / r$ by Lemma 2.2. Now the triple $\left(A^{K}, B, r C\right)$ is a stabilisable and detectable realization of the transfer function $r \mathbf{G}^{K}$ and an application of the bounded real lemma (see, for example, [17, Theorem 5.3 .25 , Remark 5.3.27, p. 604]) to $\left(A^{K}, B, r C\right)$ shows that there exist a positive semi-definite $P=P^{T} \in$ $\mathbb{R}^{n \times n}$ and $L \in \mathbb{R}^{m \times n}$ such that (A.4) holds.

Note that (A.4) implies that $\operatorname{ker} P \subseteq \operatorname{ker} C$. Obviously, $|\cdot|_{P}$ is a norm on $(\operatorname{ker} P)^{\perp}$ and so there exists $l>0$ such that $\|z\| \leq l|z|_{P}$ for all $z \in(\operatorname{ker} P)^{\perp}$. Hence, for all $z=z_{1}+z_{2} \in \mathbb{R}^{n}, z_{1} \in(\operatorname{ker} P)^{\perp}$ and $z_{2} \in \operatorname{ker} P$, we have that

$$
\begin{aligned}
\|C z\| & =\left\|C z_{1}+C z_{2}\right\|=\left\|C z_{1}\right\| \leq\|C\|\left\|z_{1}\right\| \\
& \leq\left. l\|C\||| z_{1}\right|_{P}=l\|C\||| z_{1}+\left.z_{2}\right|_{P}=d|z|_{P},
\end{aligned}
$$

where $d:=l\|C\|$, which is (A.5).

Next, since $A^{K}$ is Hurwitz, there exists positive definite $Q_{0}=Q_{0}^{T} \in \mathbb{R}^{n \times n}$ such that

$$
\left(A^{K}\right)^{T} Q_{0}+Q_{0} A^{K}=-I .
$$

It follows that

$$
\begin{aligned}
& \left\langle A^{K} z+B u, Q_{0} z\right\rangle+\left\langle z, Q_{0}\left(A^{K} z+B u\right)\right\rangle \\
= & \left\langle\left(\left(A^{K}\right)^{T} Q_{0}+Q_{0} A^{K}\right) z, z\right\rangle+2\left\langle z, Q_{0} B u\right\rangle \\
\leq & -\|z\|^{2}+2\left\|Q_{0} B\right\|\|z\|\|u\| \quad \forall(z, u) \in \mathbb{R}^{n} \times \mathbb{R}^{m} .
\end{aligned}
$$

By using the inequality

$$
2 z_{1} z_{2} \leq \rho z_{1}^{2}+z_{2}^{2} / \rho \quad \forall z_{1}, z_{2} \in \mathbb{R}, \forall \rho>0,
$$

on the second term on the right hand side of the above inequality, and defining $Q$ as a suitable positive multiple of $Q_{0}$, we conclude that there exists $\delta>0$ such that (A.6) holds. 
Proof of Theorem 3.1. The proof draws inspiration from ideas in $[2,29]$, but the actual arguments differ substantially to those used in $[2,29]$ because of the different stability notions and Lur's systems under consideration.

Statement (1). Trivially, if the inequality (3.1) is satisfied with $\alpha=\beta \in \mathcal{K}_{\infty}$, then, a fortiori, it holds for any $\alpha \in \mathcal{K} \backslash \mathcal{K}_{\infty}$ such that $\alpha(s) \leq \beta(s)$ for all $s \geq 0$. Therefore, without loss of generality, we may assume that $\alpha \in \mathcal{K} \backslash \mathcal{K}_{\infty}$, and so, $\alpha$ is bounded.

There are two claims to prove: system (1.1) is integral ISS and small-signal ISS.

Integral ISS. The inequality (3.1) implies that (2.6) holds, and so an application of Theorem 2.3 ensures that the unforced Lur'e system is 0-GAS. We seek to apply Theorem A.1 to establish iISS via a Lyapunov analysis, and so we shall construct a function $V$ satisfying (A.1) with $\zeta$ given by $\zeta(s)=a s+b s^{2}$ for all $s \geq 0$, where $a>0$ and $b \geq 0$, with $b=0$ if $D_{\mathrm{e}}=0$. Furthermore, we will establish that $\zeta$ is an iISS gain for the Lur'e system (1.1) and thus the comparison function $\gamma_{2}$ can be chosen to satisfy (3.2).

We proceed in three steps.

Step 1: Preparations For The Lyapunov AnalYSIS. The inequality (3.1) implies that

$$
\left\|f^{K}(z)\right\|^{2} \leq r^{2}\|z\|^{2} \quad \forall z \in \mathbb{R}^{p},
$$

where recall that $f^{K}$ is defined in (A.3). Moreover, it follows from (3.1) that

$$
\alpha(s) \leq r s \quad \forall s \in \mathbb{R}_{+} .
$$

Let $P$ and $Q$ be as in Lemma A.3. By equivalence of the norms $\|\cdot\|$ and $|\cdot|_{Q}$, there exist $q_{1}, q_{2}>0$ such that

$$
q_{1}\|z\| \leq|z|_{Q} \leq q_{2}\|z\| \quad \forall z \in \mathbb{R}^{n} .
$$

Furthermore, there exists $\mu>0$ such that

$$
|z|_{P} \leq \mu|z|_{Q} \quad \forall z \in \mathbb{R}^{n}
$$

Define the positive constants

$$
\left.\begin{array}{l}
j_{0}:=\delta / q_{2}^{2}, \quad j_{1}:=\min \left\{1, \sqrt{j_{0}} /(2 r)\right\}, \\
j_{2}:=\min \left\{1, \sqrt{j_{0}} /(d r)\right\},
\end{array}\right\}
$$

where $\delta$ is as in (A.6). Define $\theta: \mathbb{R}_{+} \rightarrow \mathbb{R}_{+}$by

$$
\theta(s)= \begin{cases}0 & s=0 \\ \frac{1}{s} \int_{0}^{s} \alpha\left(j_{1} \tau\right) d \tau & s>0\end{cases}
$$

The function $\theta$ is positive and continuously differentiable on $(0, \infty)$ and satisfies

$$
0 \leq \theta(s) \leq \alpha\left(j_{1} s\right) \leq \alpha(s) \quad \forall s \geq 0,
$$

and

$$
\theta(s)+s \theta^{\prime}(s)=\frac{d}{d s}(s \theta(s))=\alpha\left(j_{1} s\right) \quad \forall s>0 .
$$

The combination of (A.13) and (A.14) yields that $\theta^{\prime}(s) \geq 0$ for all $s>0$, and so $\theta$ is non-decreasing. Choose $\nu>0$ sufficiently small so that

$$
\min \left\{s^{2}, \frac{1}{1+s}\right\}=s^{2} \quad \forall s \in\left[0, \frac{r^{3} \nu^{2}}{j_{0}}\right] .
$$

Since the function $s \mapsto s \theta\left(j_{2} s\right)$ is in $\mathcal{K}_{\infty}$, it is clear that there exists $\varepsilon_{1}>0$ such that

$$
\begin{array}{r}
\frac{\varepsilon_{1} r|C|_{Q} \mu\|\alpha\|_{L^{\infty}}}{q_{1}} s^{3} \leq 1 \text { for all } s \geq 0 \text { such that } \\
\\
s \theta\left(j_{2} s\right) \leq r^{3} \nu^{2} / j_{0} .
\end{array}
$$

Choose $\varepsilon_{2}>0$ sufficiently small so that

$$
\begin{aligned}
& \varepsilon_{2} \min \left\{s^{2} \theta^{2}\left(j_{2} s\right), \frac{1}{1+s \theta\left(j_{2} s\right)}\right\} s \alpha\left(j_{1} j_{2} s\right) \\
\leq & \frac{q_{1} \alpha(\nu / 2) \alpha\left(j_{1} \nu / d\right)}{r|C|_{Q} \mu} \quad \forall s \geq 0 .
\end{aligned}
$$

Such a choice is possible owing to the boundedness of $\alpha$ and the fact that $\theta$ is non-decreasing. Set $\varepsilon:=$ $\min \left\{\varepsilon_{1}, \varepsilon_{2}\right\}$ and define $k, h: \mathbb{R}_{+} \rightarrow \mathbb{R}_{+}$by

$$
\left.\begin{array}{rl}
k(s) & =\varepsilon \min \left\{s^{2}, \frac{1}{1+s}\right\} \\
\text { and } \quad h(s) & =\int_{0}^{s} k(\tau) d \tau
\end{array}\right\} \text { for all } s \geq 0 .
$$

Step 2: Lyapunov Analysis. Define $V: \mathbb{R}^{n} \rightarrow \mathbb{R}_{+}$ by

$$
V(z):=2|z|_{P} \theta\left(|z|_{P}\right)+2 h\left(|z|_{Q} \theta\left(j_{2}|z|_{Q}\right)\right) \quad \forall z \in \mathbb{R}^{n} .
$$

This function is continuously differentiable and satisfies $V(0)=0$ and $V(z)>0$ for $z \neq 0$. Moreover, by setting

$$
\left.\begin{array}{rl}
V_{P}(z) & :=2|z|_{P} \theta\left(|z|_{P}\right) \\
\text { and } \quad V_{Q}(z) & :=2 h\left(|z|_{Q} \theta\left(j_{2}|z|_{Q}\right)\right)
\end{array}\right\} \quad \forall z \in \mathbb{R}^{n},
$$

it is clear that

$$
V(z) \geq V_{Q}(z)=2 h\left(|z|_{Q} \theta\left(j_{2}|z|_{Q}\right)\right) \quad \forall z \in \mathbb{R}^{n},
$$

and so $V$ is radially unbounded as

$$
h(s)=\int_{0}^{s} k(\tau) d \tau \geq \varepsilon \int_{1}^{s} \frac{1}{1+\tau} d \tau \quad \forall s \geq 1,
$$

diverges as $s \rightarrow \infty$.

Defining the map $F: \mathbb{R}^{n} \times \mathbb{R}^{q} \rightarrow \mathbb{R}^{n}$ by

$$
F(z, w):=A^{K} z+B f^{K}\left(C z+D_{\mathrm{e}} w\right)+B_{\mathrm{e}}^{K} w,
$$

it is clear that, for all $(z, w) \in \mathbb{R}^{n} \times \mathbb{R}^{q}$,

$$
F(z, w)=A z+B f\left(C z+D_{\mathrm{e}} w\right)+B_{\mathrm{e}} w .
$$

We will show that there exists $c>0$ such that

$$
\begin{array}{r}
\langle(\nabla V)(z), F(z, w)\rangle \leq c\left(\left\|B_{\mathrm{e}}^{K} w\right\|+\left\|D_{\mathrm{e}} w\right\|+\left\|D_{\mathrm{e}} w\right\|^{2}\right) \\
\forall(z, w) \in \mathbb{R}^{n} \times \mathbb{R}^{q} .(\mathrm{A} .20)
\end{array}
$$


Setting $y=C z+D_{\mathrm{e}} w$, a straightforward calculation using (A.14) shows that

$$
\begin{gathered}
\left\langle\left(\nabla V_{P}\right)(z), F(z, w)\right\rangle \\
=2\left\langle P z, A^{K} z+B f^{K}(y)+B_{\mathrm{e}}^{K} w\right\rangle \frac{\alpha\left(j_{1}|z|_{P}\right)}{|z|_{P}} \\
\forall(z, w) \in \mathbb{R}^{n} \times \mathbb{R}^{q} \text { with } z \notin \operatorname{ker} P .
\end{gathered}
$$

Using the Cauchy-Schwarz inequality, we majorise the terms in (A.21) which contain $B_{\mathrm{e}}^{K} w$ by

$$
\begin{aligned}
2\left\langle P z, B_{\mathrm{e}}^{K} w\right\rangle \frac{\alpha\left(j_{1}|z|_{P}\right)}{|z|_{P}} & \leq 2 \alpha\left(j_{1}|z|_{P}\right)\left|B_{\mathrm{e}}^{K} w\right|_{P} \\
& \leq c_{1}\left\|B_{\mathrm{e}}^{K} w\right\|,
\end{aligned}
$$

where $c_{1}:=2 \sqrt{\|P\|}\|\alpha\|_{L^{\infty}}$. Next, we estimate the terms in (A.21) which do not contain $B_{\mathrm{e}}^{K} w$. Using (A.4), we compute

$$
\begin{aligned}
& 2\left\langle P z, A^{K} z+B f^{K}(y)\right\rangle \\
&=-r^{2}\|C z\|^{2}-\left\|L z+f^{K}(y)\right\|^{2}+\left\|f^{K}(y)\right\|^{2} \\
& \leq-r^{2}\|C z\|^{2}+\left\|f^{K}(y)\right\|^{2} \\
&=-r^{2}\|y\|^{2}+\left\|f^{K}(y)\right\|^{2}+r^{2}\left\|D_{\mathrm{e}} w\right\|^{2}+2 r^{2}\left\langle C z, D_{\mathrm{e}} w\right\rangle \\
& \leq-r\|y\| \alpha(\|y\|)+r^{2}\left\|D_{\mathrm{e}} w\right\|^{2}+2 r^{2}\left\langle C z, D_{\mathrm{e}} w\right\rangle \\
& \quad \forall(z, w) \in \mathbb{R}^{n} \times \mathbb{R}^{q},
\end{aligned}
$$

where the last inequality follows from (3.1) and (A.9). Using (A.5) and (A.9), we obtain that

$$
\begin{gathered}
\left(\left\|D_{\mathrm{e}} w\right\|^{2}+2\left\langle C z, D_{\mathrm{e}} w\right\rangle\right) \frac{\alpha\left(j_{1}|z|_{P}\right)}{|z|_{P}} \\
\leq \max \left\{r j_{1}, 2 d\|\alpha\|_{L^{\infty}}\right\}\left(\left\|D_{\mathrm{e}} w\right\|+\left\|D_{\mathrm{e}} w\right\|^{2}\right) \\
\forall(z, w) \in \mathbb{R}^{n} \times \mathbb{R}^{q}, z \notin \operatorname{ker} P .
\end{gathered}
$$

Since $\alpha$ is non-negative, we obtain from (A.23) and (A.24) that

$$
\begin{gathered}
2\left\langle P z, A^{K} z+B f^{K}(y)\right\rangle \frac{\alpha\left(j_{1}|z|_{P}\right)}{|z|_{P}} \\
\leq-r\|y\| \alpha(\|y\|) \frac{\alpha\left(j_{1}|z|_{P}\right)}{|z|_{P}}+c_{2}\left(\left\|D_{\mathrm{e}} w\right\|+\left\|D_{\mathrm{e}} w\right\|^{2}\right) \\
\forall(z, w) \in \mathbb{R}^{n} \times \mathbb{R}^{q}, z \notin \operatorname{ker} P,
\end{gathered}
$$

where $c_{2}:=r^{2} \max \left\{r j_{1}, 2 d\|\alpha\|_{L^{\infty}}\right\}$. Therefore, the conjunction of (A.21), (A.22) and (A.25) gives,

$$
\begin{aligned}
& \left\langle\left(\nabla V_{P}\right)(z), F(z, w)\right\rangle \\
\leq & -r\|y\| \alpha(\|y\|) \frac{\alpha\left(j_{1}|z|_{P}\right)}{|z|_{P}}+c_{1}\left\|B_{\mathrm{e}}^{K} w\right\|+c_{2}\left(\left\|D_{\mathrm{e}} w\right\|\right. \\
& \left.+\left\|D_{\mathrm{e}} w\right\|^{2}\right) \forall(z, w) \in \mathbb{R}^{n} \times \mathbb{R}^{q}, z \notin \operatorname{ker} P .
\end{aligned}
$$

Next we estimate the inner product $\left\langle\left(\nabla V_{Q}\right)(z), F(z, w)\right\rangle$. To this end, set $k_{z}:=$ $k\left(|z|_{Q} \theta\left(j_{2}|z|_{Q}\right)\right)$ for $z \in \mathbb{R}^{n}$. A calculation simi- lar to that leading to (A.21) yields

$$
\begin{aligned}
&\left\langle\left(\nabla V_{Q}\right)(z), F(z, w)\right\rangle \\
&= 2 h^{\prime}\left(|z|_{Q} \theta\left(j_{2}|z|_{Q}\right)\right)\langle F(z, w), Q z\rangle \frac{\alpha\left(j_{1} j_{2}|z|_{Q}\right)}{|z|_{Q}} \\
&=2 k_{z}\left\langle A^{K} z+B f^{K}(y)+B_{\mathrm{e}}^{K} w, Q z\right\rangle \frac{\alpha\left(j_{1} j_{2}|z|_{Q}\right)}{|z|_{Q}} \\
& \forall(z, w) \in \mathbb{R}^{n} \times \mathbb{R}^{q}, z \neq 0, \quad \mathrm{~A} .
\end{aligned}
$$

where we have used that $h^{\prime}=k$. We estimate the terms on the right hand side of (A.27) with and without $B_{\mathrm{e}}^{K} w$ separately. For which purpose, we invoke the Cauchy-Schwarz inequality, to obtain, for all $(z, w) \in \mathbb{R}^{n} \times \mathbb{R}^{q}$ with $z \neq 0$

$$
\begin{aligned}
2 k_{z}\left\langle B_{\mathrm{e}}^{K} w, Q z\right\rangle \frac{\alpha\left(j_{1} j_{2}|z|_{Q}\right)}{|z|_{Q}} & \leq 2\|k\|_{L^{\infty}}\|\alpha\|_{L^{\infty}\left|B_{\mathrm{e}}^{K} w\right|_{Q}} \\
& =c_{3}\left\|B_{\mathrm{e}}^{K} w\right\|,
\end{aligned}
$$

where $c_{3}:=2 q_{2}\|k\|_{L^{\infty}}\|\alpha\|_{L^{\infty}}$ and $q_{2}$ is as in (A.10). Returning to (A.27), we now use (A.6) and (A.8) to estimate that

$$
\begin{aligned}
& 2\left\langle A^{K} z+B f^{K}(y), Q z\right\rangle \\
& \leq-\delta\|z\|^{2}+r^{2}\left(\|C z\|^{2}+2\|C z\|\left\|D_{\mathrm{e}} w\right\|+\left\|D_{\mathrm{e}} w\right\|^{2}\right) \\
& \quad \forall(z, w) \in \mathbb{R}^{n} \times \mathbb{R}^{q} .
\end{aligned}
$$

Akin to (A.24), we estimate using (A.5) and (A.9) that

$$
\begin{gathered}
r^{2} k_{z}\left(2\|C z\|\left\|D_{\mathrm{e}} w\right\|+\left\|D_{\mathrm{e}} w\right\|^{2}\right) \frac{\alpha\left(j_{1} j_{2}|z|_{Q}\right)}{|z|_{Q}} \\
\leq r^{2}\|k\|_{L^{\infty}} \max \left\{2 d \mu\|\alpha\|_{L^{\infty}}, r j_{1} j_{2}\right\}\left(\left\|D_{\mathrm{e}} w\right\|+\left\|D_{\mathrm{e}} w\right\|^{2}\right) \\
\forall(z, w) \in \mathbb{R}^{n} \times \mathbb{R}^{q}, z \neq 0, \quad(\mathrm{~A} .30)
\end{gathered}
$$

where we have made use of (A.11). Combining (A.27)(A.30) yields that, for all $(z, w) \in \mathbb{R}^{n} \times \mathbb{R}^{q}$ with $z \neq 0$

$$
\begin{aligned}
& \left\langle\left(\nabla V_{Q}\right)(z), F(z, w)\right\rangle \\
\leq & k_{z}\left(-\delta\|z\|^{2}+r^{2}\|C z\|^{2}\right) \frac{\alpha\left(j_{1} j_{2}|z|_{Q}\right)}{|z|_{Q}}+c_{3}\left\|B_{\mathrm{e}}^{K} w\right\| \\
& +c_{4}\left(\left\|D_{\mathrm{e}} w\right\|+\left\|D_{\mathrm{e}} w\right\|^{2}\right),
\end{aligned}
$$

where $c_{4}:=r^{2}\|k\|_{L^{\infty}} \max \left\{2 d \mu\|\alpha\|_{L^{\infty}}, r j_{1} j_{2}\right\}$. We claim that there exists $c_{5}>0$ such that,

$$
\begin{gathered}
r^{2}\|C z\|^{2} k_{z} \frac{\alpha\left(j_{1} j_{2}|z|_{Q}\right)}{|z|_{Q}} \leq r\|y\| \alpha(\|y\|) \frac{\alpha\left(j_{1}|z|_{P}\right)}{|z|_{P}} \\
+j_{0} k_{z}|z|_{Q} \alpha\left(j_{1} j_{2}|z|_{Q}\right)+c_{5}\left\|D_{\mathrm{e}} w\right\| \\
\forall(z, w) \in \mathbb{R}^{n} \times \mathbb{R}^{q}, z \notin \operatorname{ker} P .
\end{gathered}
$$

So as to avoid disrupting the flow of the present argument, we postpone the proof of (A.32) until later.

By choice of the constant $j_{0}>0$ we have that

$$
\begin{array}{r}
j_{0} k_{z}|z|_{Q} \alpha\left(j_{1} j_{2}|z|_{Q}\right) \leq \delta\|z\|^{2} k_{z} \frac{\alpha\left(j_{1} j_{2}|z|_{Q}\right)}{|z|_{Q}} \\
\forall z \in \mathbb{R}^{n}, z \neq 0 .
\end{array}
$$


Adding (A.26) and (A.31), and using (A.32) and (A.33), we arrive at the estimate

$$
\begin{aligned}
\langle(\nabla V)(z), F(z, w)\rangle & =\left\langle\left(\nabla V_{P}+\nabla V_{Q}\right)(z), F(z, w)\right\rangle \\
\leq & c\left(\left\|B_{\mathrm{e}}^{K} w\right\|+\left\|D_{\mathrm{e}} w\right\|+\left\|D_{\mathrm{e}} w\right\|^{2}\right) \\
\forall(z, w) & \in \mathbb{R}^{n} \times \mathbb{R}^{q}, z \notin \operatorname{ker} P, \quad(\mathrm{~A} .34)
\end{aligned}
$$

where $c:=\sum_{i=1}^{5} c_{i}$. Let now $z \in \operatorname{ker} P$. Choosing $z^{\perp} \in(\operatorname{ker} P)^{\perp}, z^{\perp} \neq 0$ (which is possible since $P \neq 0$ ), we have that $z_{\lambda}:=z+\lambda z^{\perp} \notin$ ker $P$ for every non-zero $\lambda \in \mathbb{R}$. For $w \in \mathbb{R}^{q}$, it follows from (A.34) that

$$
\begin{gathered}
\left\langle(\nabla V)\left(z_{\lambda}\right), F\left(z_{\lambda}, w\right)\right\rangle \leq c\left(\left\|B_{\mathrm{e}}^{K} w\right\|+\left\|D_{\mathrm{e}} w\right\|+\left\|D_{\mathrm{e}} w\right\|^{2}\right) \\
\forall w \in \mathbb{R}^{q}, \forall \lambda \in \mathbb{R}, \quad \lambda \neq 0 .
\end{gathered}
$$

Letting $\lambda \rightarrow 0$ above and invoking the continuity of $\nabla V$ and $F$, we infer that (A.34) extends to all $(z, w) \in$ $\mathbb{R}^{n} \times \mathbb{R}^{q}$, which yields (A.20). Therefore, setting

$$
a:=c\left(\left\|B_{\mathrm{e}}^{K}\right\|+\left\|D_{\mathrm{e}}\right\|\right) \quad \text { and } \quad b:=c\left\|D_{\mathrm{e}}\right\|^{2},
$$

and $\zeta(s):=a s+b s^{2}$, we arrive at, for all $(z, w) \in$ $\mathbb{R}^{n} \times \mathbb{R}^{q}$

$$
\langle(\nabla V)(z), F(z, w)\rangle \leq \zeta(\|w\|) .
$$

It now follows from Theorem A.1 and (A.19) that the Lur'e system (1.1) is iISS.

Step 3: The Function $\gamma_{2}$ In (2.4) MAY BE CHOSEN TO SATISFY (3.2). We note that, for every compact set $\Gamma \subset \mathbb{R}^{n}$, there exists $l>0$ such that

$$
\|F(z, w)\| \leq l(1+\zeta(\|w\|)) \quad \forall(z, w) \in \Gamma \times \mathbb{R}^{q} .
$$

Together with (A.35) and [42, Theorem 2.4] this implies that (2.4) holds with $\gamma_{2}=\zeta$, showing that $\gamma_{2}$ may be chosen to satisfy (3.2). Moreover, if $D_{\mathrm{e}}=0$, then $b=0$, in which case $\gamma_{2}=\zeta$ is linear.

To complete the argument which will establish the iISS property, it remains to show that (A.32) holds. To this end, we first prove that

$$
\begin{aligned}
k_{z} r^{2}\|C z\|^{2} \frac{\alpha\left(j_{1} j_{2}|z|_{Q}\right)}{|z|_{Q}} \leq & r\|C z\| \alpha(\|C z\| / 2) \frac{\alpha\left(j_{1}|z|_{P}\right)}{|z|_{P}} \\
& +j_{0} k_{z}|z|_{Q}^{2} \frac{\alpha\left(j_{1} j_{2}|z|_{Q}\right)}{|z|_{Q}} \\
& \forall z \in \mathbb{R}^{n} \backslash \operatorname{ker} P .
\end{aligned}
$$

Since (A.36) trivially holds if $C z=0$, we assume that $C z \neq 0$ and consider two exhaustive cases.

CASE 1: $\|C z\| \geq \nu$, where $\nu>0$ is as in (A.15). In this case, it follows from (A.5) that

$$
\nu \leq d|z|_{P}
$$

and so, we may estimate

$$
\begin{aligned}
k_{z} r\|C z\| \frac{\alpha\left(j_{1} j_{2}|z|_{Q}\right)}{|z|_{Q}}|z|_{P} & \leq \alpha(\nu / 2) \alpha\left(j_{1} \nu / d\right) \\
& \leq \alpha(\|C z\| / 2) \alpha\left(j_{1}|z|_{P}\right)
\end{aligned}
$$

where the first inequality follows from (A.17) and the definition of $k$, whilst we have used (A.37) and the fact that $\alpha$ is increasing to obtain the second inequality. Dividing both sides of the above by $|z|_{P}>0$ and multiplying by $r\|C z\|$ gives (A.36), as required.

CAse 2: $\|C z\|<\nu$. We note that if

$$
r^{2}\|C z\|^{2} \leq j_{0}|z|_{Q}^{2},
$$

then (A.36) holds, because in this case the LHS of (A.36) is less or equal to the second term on the RHS of (A.36). Therefore, we assume that

$$
j_{0}|z|_{Q}^{2} \leq r^{2}\|C z\|^{2} .
$$

Hence, $j_{0}|z|_{Q}^{2} \leq r^{2}\|C z\|^{2} \leq r^{2} \nu^{2}$, and so,

$$
|z|_{Q} \theta\left(j_{2}|z|_{Q}\right) \leq|z|_{Q} \alpha\left(j_{1} j_{2}|z|_{Q}\right) \leq \frac{r^{3} \nu^{2}}{j_{0}},
$$

where the first inequality follows from (A.9), (A.13), and the fact that $j_{1}, j_{2} \leq 1$. Consequently, in light of (A.15), we have that, for all $z$ satisfying (A.38),

$$
\begin{aligned}
k\left(|z|_{Q} \theta\left(j_{2}|z|_{Q}\right)\right) & =\varepsilon|z|_{Q}^{2} \theta^{2}\left(j_{2}|z|_{Q}\right) \\
& \leq \varepsilon_{1}|z|_{Q}^{2} \theta^{2}\left(j_{2}|z|_{Q}\right) .
\end{aligned}
$$

We require two further estimates. The first,

$$
\begin{aligned}
\theta\left(j_{2}|z|_{Q}\right) & \leq \alpha\left(j_{1} j_{2}|z|_{Q}\right) \leq \alpha\left(j_{1}|z|_{Q}\right) \\
& \leq \alpha\left(j_{1}\left(r / \sqrt{j_{0}}\right)\|C z\|\right) \leq \alpha(\|C z\| / 2),
\end{aligned}
$$

follows from (A.12) and (A.13) and the monotonicity of $\alpha$. The second,

$$
\begin{aligned}
\theta\left(j_{2}|z|_{Q}\right) & \leq \alpha\left(j_{1} j_{2}|z|_{Q}\right) \leq \alpha\left(j_{1} j_{2}\left(r / \sqrt{j_{0}}\right)\|C z\|\right) \\
& \leq \alpha\left(j_{1}\left(j_{2} r d / \sqrt{j_{0}}\right)|z|_{P}\right) \leq \alpha\left(j_{1}|z|_{P}\right)
\end{aligned}
$$

is a consequence of (A.5), (A.12) and (A.13).

Appealing to (A.39)-(A.42), we obtain that

$$
\begin{aligned}
& k\left(|z|_{Q} \theta\left(j_{2}|z|_{Q}\right)\right) r\|C z\| \frac{\alpha\left(j_{1} j_{2}|z|_{Q}\right)}{|z|_{Q}}|z|_{P} \\
\leq & \varepsilon_{1}|z|_{Q}^{2} \theta^{2}\left(j_{2}\|z\|_{Q}\right) r\|C z\| \frac{\alpha\left(j_{1} j_{2}|z|_{Q}\right)}{|z|_{Q}}|z|_{P} \\
\leq & \varepsilon_{1} r|z|_{Q}^{2}\|C z\| \alpha(\|C z\| / 2) \alpha\left(j_{1}|z|_{P}\right) \frac{\alpha\left(j_{1} j_{2}|z|_{Q}\right)}{|z|_{Q}}|z|_{P} \\
\leq & \frac{\varepsilon_{1} r|C|_{Q} \mu\|\alpha\|_{L^{\infty}}}{q_{1}}|z|_{Q}^{3} \alpha(\|C z\| / 2) \alpha\left(j_{1}|z|_{P}\right) \\
\leq & \alpha(\|C z\| / 2) \alpha\left(j_{1}|z|_{P}\right)
\end{aligned}
$$

where the final inequality above follows from the definition of $\varepsilon_{1}$ in (A.16) and crucially uses (A.39). Dividing both sides of (A.43) by $|z|_{P}>0$ and multiplying by $r\|C z\|$ gives (A.36).

Using that $y=C z+D_{\mathrm{e}} w$, and $\alpha$ is increasing and bounded, it is routine to prove that

$$
\begin{array}{r}
\|C z\| \alpha(\|C z\| / 2) \leq\|y\| \alpha(\|y\|)+2\|\alpha\|_{L^{\infty}}\left\|D_{\mathrm{e}} w\right\| \\
\forall(z, w) \in \mathbb{R}^{n} \times \mathbb{R}^{q} .
\end{array}
$$


Inserting (A.44) into (A.36) yields (A.32) with $c_{5}:=$ $2\|\alpha\|_{L^{\infty}} r j_{1}$, where we have used that $\alpha\left(j_{1}|z|_{P}\right) \leq$ $r j_{1}|z|_{P}$ for all $z \in \mathbb{R}^{n}$.

Small-signal ISS. The proof is very similar to that of [29, Theorem 3.2], and so we only outline the argument. To establish the small-signal ISS property, it suffices to find $R>0$, a continuously differentiable $W: \mathbb{R}^{n} \rightarrow \mathbb{R}_{+}$, and $\alpha_{1}, \alpha_{2}, \gamma, \mu \in \mathcal{K}_{\infty}$ such that

$$
\alpha_{1}(\|z\|) \leq W(z) \leq \alpha_{2}(\|z\|) \quad \forall z \in \mathbb{R}^{n},
$$

and, furthermore,

$$
\begin{aligned}
\langle(\nabla W)(z), F(z, w)\rangle \leq & -\gamma(\|z\|)+\mu(\|w\|) \\
& \forall(z, w) \in \mathbb{R}^{n} \times B_{R}(0),
\end{aligned}
$$

see [6, Section IV.A.], where $B_{R}(0)$ is the open ball in $\mathbb{R}^{q}$ centred at the origin and of radius $R$. Let $P, Q \in$ $\mathbb{R}^{n \times n}$ be as in Lemma A.3, and satisfy (A.4) and (A.6), respectively. A suitable $W$ is given by

$$
W(z):=|z|_{P}^{2}+h\left(|z|_{Q}^{2}\right) \quad \forall z \in \mathbb{R}^{n},
$$

where $h: \mathbb{R}_{+} \rightarrow \mathbb{R}_{+}$is defined by $h(0)=0$ and

$$
h(s)=\nu_{0} \int_{0}^{s} \min \left\{\tau, \frac{\alpha\left(\nu_{1} \sqrt{\tau}\right)}{\sqrt{\tau}}\right\} d \tau \quad \forall s>0,
$$

with $\nu_{0}:=q_{1} /(r\|C\|)$ and $\nu_{1}:=\sqrt{\delta} /\left(4 r q_{2}\right)$. The motivation for the choice of $\nu_{0}$ and $\nu_{1}$ is apparent when estimating $\langle\nabla W(z), F(z, w)\rangle$. The argument now mirrors that used in the proof of [29, Theorem 3.2]. The function $\mu$ defined on $[29$, p.448] is replaced by the $\mathcal{K}$ function

$$
\kappa(s):=\min \left\{\nu_{2} s^{3}, \nu_{3} \alpha\left(\nu_{1} q_{1} s\right)\right\} \quad \forall s \geq 0,
$$

where $\nu_{2}:=\left(\nu_{0} q_{1}^{2} \delta\right) / 8$ and $\nu_{3}:=\nu_{0} \delta /\left(8 q_{2}\right)$, and references to [29, Lemma 2.4] should be replaced by references to Lemma A.2. Although [29, Theorem 3.2] considers the case wherein $D_{\mathrm{e}}=0$, the analysis in [29] extends to the case of nonzero $D_{\mathrm{e}}$ (which gives rise to extra terms of the form $\left\|D_{\mathrm{e}} w\right\|^{2}$, cf. (A.26) and (A.31)) and shows that there exist constants $d, R>0$ such that, for all $(z, w) \in \mathbb{R}^{n} \times B_{R}(0)$,

$$
\langle\nabla W(z), F(z, w)\rangle \leq-\|z\| \kappa(\|z\|)+d\left(\|w\|+\|w\|^{2}\right) .
$$

The proof of statement (1) is complete.

Statement (2). This statement is a generalisation of $\left[29\right.$, Theorem 3.2] to the case wherein $D_{\text {e }} \neq 0$. The proof in [29] extends to this case subject to comments similar to those made in the proof of the small-signal ISS property. In particular, since $\alpha \in \mathcal{K}_{\infty}$, the function $\kappa$ in (A.48) is in $\mathcal{K}_{\infty}$, and $W$ given in (A.47) is an ISS Lyapunov function for (1.1), completing the proof of the theorem.

\section{A.2 Remaining proofs}

Proof of Corollary 3.4. We rewrite (1.1) as

$$
\begin{aligned}
\dot{x}= & A x+B f(C x)+B\left(f\left(C x+D_{\mathrm{e}} v\right)-f(C x)\right) \\
& +B_{\mathrm{e}} v, \quad x(0)=x^{0},
\end{aligned}
$$

which we view as another instance of (1.1) with $B_{\mathrm{e}}$, $D_{\mathrm{e}}$ and $v$ replaced by

$$
\begin{aligned}
\tilde{B}_{\mathrm{e}} & :=\left(\begin{array}{cc}
B_{\mathrm{e}} & I
\end{array}\right), \quad \tilde{D}_{\mathrm{e}}:=0, \\
\text { and } \quad \tilde{v} & :=\left(\begin{array}{c}
v \\
f\left(C x+D_{\mathrm{e}} v\right)-f(C x)
\end{array}\right),
\end{aligned}
$$

respectively. An application of statement (1) of Theorem 3.1 to (A.49) shows that (A.49) is iISS (with respect to the input $\tilde{v}$ ) and, as $\tilde{D}_{\mathrm{e}}=0, \gamma_{2}$ is of the form $\gamma_{2}(s)=a s$ for some constant $a>0$. The claim now follows since, as a consequence of the global Lipschitz property, there exists $\lambda>0$ such that $\|\tilde{v}(t)\| \leq \lambda\|v(t)\|$ for all $t \geq 0$.

Proof of Corollary 3.6. We claim that

$$
\left\|\mathbf{G}^{K}\right\|_{H^{\infty}} \leq 1 / r .
$$

If (A.50) holds, then Lemma 2.2 yields that $\mathbb{B}_{\mathbb{C}}(K, r) \subseteq \mathbb{S}_{\mathbb{C}}(\mathbf{G})$, and the corollary now follows from Theorem 3.1.

It remains to prove (A.50). Seeking a contradiction, suppose that $\left\|\mathbf{G}^{K}\right\|_{H^{\infty}}>1 / r$. By the real supremum value property, there exists $s_{*} \in\{s \in \mathbb{C}: \operatorname{Re} s \geq$ $0\} \cup\{\infty\}$ such that $\mathbf{G}^{K}\left(s_{*}\right) \in \mathbb{R}^{p \times m}$ and

$$
\left\|\mathbf{G}^{K}\left(s_{*}\right)\right\|=\left\|\mathbf{G}^{K}\right\|_{H^{\infty}}>\frac{1}{r} .
$$

Note that here $\left\|\mathbf{G}^{K}\left(s_{*}\right)\right\|$ is the 2-norm induced norm of $\mathbf{G}^{K}\left(s_{*}\right)$ as an operator from $\mathbb{C}^{m}$ to $\mathbb{C}^{p}$. Since, for $R \in \mathbb{R}^{p \times m}$, the real and complex operator norms induced by the 2-norm coincide [40], that is,

$$
\sup _{x \in \mathbb{R}^{m}, x \neq 0} \frac{\|R x\|}{\|x\|}=\sup _{z \in \mathbb{C}^{m}, z \neq 0} \frac{\|R z\|}{\|z\|},
$$

there exists $u \in \mathbb{R}^{m}$ such that $\|u\|=1$ and

$$
\left\|\mathbf{G}^{K}\left(s_{*}\right) u\right\|=\left\|\mathbf{G}^{K}\left(s_{*}\right)\right\|=\left\|\mathbf{G}^{K}\right\|_{H^{\infty}} .
$$

Set $\gamma:=\left\|\mathbf{G}^{K}\left(s_{*}\right) u\right\|=\left\|\mathbf{G}^{K}\right\|_{H^{\infty}}>1 / r$, and define $w:=(1 / \gamma) \mathbf{G}^{K}\left(s_{*}\right) u \in \mathbb{R}^{p}$ and $L \in \mathbb{R}^{m \times p}$ by $L y:=$ $\langle y, w\rangle u / \gamma$ for all $y \in \mathbb{R}^{p}$. We see that

$$
\left(I-L \mathbf{G}^{K}\left(s_{*}\right)\right) u=u-L \mathbf{G}^{K}\left(s_{*}\right) u=0,
$$

so $L \notin \mathbb{S}_{\mathbb{R}}\left(\mathbf{G}^{K}\right)$, and, moreover,

$$
\|L y\|=\frac{|\langle y, w\rangle|}{\gamma}\|u\| \leq \frac{\|y\|}{\gamma}<r\|y\| \quad \forall y \in \mathbb{R}^{p},
$$

from which we deduce that $L \in \mathbb{B}_{\mathbb{R}}(0, r)$. We conclude that $\mathbb{B}_{\mathbb{R}}(0, r) \nsubseteq \mathbb{S}_{\mathbb{R}}\left(\mathbf{G}^{K}\right)$, showing that $\mathbb{B}_{\mathbb{R}}(K, r) \nsubseteq$ $\mathbb{S}_{\mathbb{R}}(\mathbf{G})$, and thus yielding a contradiction. Consequently, (A.50) holds.

Proof of Corollary 3.8. The proof is very similar to that of [29, Corollary 3.10]. Therefore, we will be brief and focus on the modifications required to make the proof of [29, Corollary 3.10] carry over to the current situation. Setting $L:=\left(K_{1}-K_{2}\right) / 2$, it can be shown 
as in [29] that $L$ is left invertible (with left inverse $\left.L^{\sharp}:=\left(L^{T} L\right)^{-1} L^{T}\right)$ and, furthermore,

$$
\mathbb{B}_{\mathbb{C}}\left(-L L^{\sharp}, 1\right) \subseteq \mathbb{S}_{\mathbb{C}}\left(L \mathbf{G}^{K_{1}}\right) .
$$

Set $A^{K_{1}}:=A+B K_{1} C$. Since $(A, B, C)$ is stabilisable and dectectable and $L$ is left invertible, it follows that the triple $\left(A^{K_{1}}, B, L C\right)$ is stabilisable and dectectable. Note that $L \mathbf{G}^{K_{1}}$ is the transfer function of $\left(A^{K_{1}}, B, L C\right)$. Defining $\tilde{f}: \mathbb{R}^{m} \rightarrow \mathbb{R}^{m}$ by

$$
\tilde{f}(\xi)=f\left(L^{\sharp} \xi\right)-K_{1} L^{\sharp} \xi \quad \forall \xi \in \mathbb{R}^{m},
$$

it is straightforward to show that $(v, x)$ satisfies (1.1) if, and only if, $(v, x)$ satisfies

$$
\begin{aligned}
\dot{x}=A^{K_{1}} x+B \tilde{f}\left(L C x+L D_{\mathrm{e}} v\right) & \\
& +\left(B_{\mathrm{e}}+B K_{1} D_{\mathrm{e}}\right) v, \quad x(0)=x^{0} .
\end{aligned}
$$

As in the proof of [29, Corollary 3.10], it can be shown that there exists $\beta \in \mathcal{K}$ such that

$$
\left\|\tilde{f}(z)+L L^{\sharp} z\right\| \leq\|z\|-\beta(\|z\|) \quad \forall z \in \mathbb{R}^{m},
$$

with $\beta \in \mathcal{K}_{\infty}$ if $\alpha \in \mathcal{K}_{\infty}$. In light of (A.51) and (A.53), an application of Theorem 3.1 (with $r=1$ and $K=$ $\left.-L L^{\sharp}\right)$ to (A.52) establishes the claim.

Proof of Proposition 3.10. We seek to invoke Theorem A.1. The 0-GAS property follows from Theorem 2.3. It remains to establish the estimate (A.1) for a suitable function $V$. To this end, set $A^{K}:=$ $A+B K C$ and let $P$ be as in (A.4). Note that the controllability/observability assumption implies that $P$ is positive definite (if the observability assumption holds, then this follows from [17, Remark 5.6.24], and a duality argument can be used to deal with the case wherein the controllability assumption is satisfied). Set

$$
\left.\begin{array}{rl}
k(s) & :=\min \left\{s, \frac{1}{1+s}\right\} \\
\text { and } \quad h(s) & :=\int_{0}^{s} k(\tau) d \tau
\end{array}\right\} \quad \forall s \geq 0,
$$

and define $V: \mathbb{R}^{n} \rightarrow \mathbb{R}_{+}$by $V(z):=h\left(|z|_{P}^{2}\right)$ for all $z \in \mathbb{R}^{n}$. It is easily seen that $V$ is continuously differentiable, radially unbounded, $V(0)=0$ and $V(z)>0$ for all $z \neq 0$ (where we have used that $P$ is positive definite). Defining $F: \mathbb{R}^{n} \times \mathbb{R}^{q} \rightarrow \mathbb{R}^{n}$ by (A.18), a routine calculation similar to that leading to (A.23) shows that

$$
\begin{gathered}
\langle(\nabla V)(z), F(z, w)\rangle=2 h^{\prime}\left(|z|_{P}^{2}\right)\langle P z, F(z, w)\rangle \\
\leq k\left(|z|_{P}^{2}\right) r^{2}\left\|D_{\mathrm{e}} w\right\|^{2}+2 k\left(|z|_{P}^{2}\right)|z|_{P}\left(r^{2} d\left\|D_{\mathrm{e}} w\right\|\right. \\
\left.+\left\|P^{1 / 2}\right\|\left\|B_{\mathrm{e}}^{K} w\right\|\right) \forall(z, w) \in \mathbb{R}^{n} \times \mathbb{R}^{q},
\end{gathered}
$$

where $d>0$ is as in (A.5). Using that the functions $k$ and $s \mapsto k\left(s^{2}\right) s$ are bounded, and appealing to (A.19), it follows that (A.1) holds with $\zeta$ given by $\zeta(s)=$ $a s+b s^{2}$, where $a>0$ and $b \geq 0$ are suitable constants. The argument that $\zeta$ is an iISS gain is the same as that in the proof of Theorem 3.1. Consequently, $\zeta$ is a possible choice for $\gamma_{2}$, showing that $\gamma_{2}$ may be chosen to satisfy (3.2).
Proof of Theorem 4.2: We seek to apply statement (1) of Theorem 3.1 with $r:=\|K\|>0$ and $f: \mathbb{R}^{p} \rightarrow$ $\mathbb{R}^{m}$ given by $f(z):=g(K z)$. For which purpose, it only remains to check that there exists $\alpha \in \mathcal{K}$ such that (3.1) holds. Since $g \in \mathcal{F}$, there exist $\beta, \gamma, \delta>0$ such that properties $(\mathcal{F} .1)$ and $(\mathcal{F} .2)$ hold.

Let $z \in \mathbb{R}^{p}$. Invoking $(\mathcal{F} .1)$, we estimate that

$$
\begin{aligned}
\|f(z)-K z\|^{2} & =\|g(K z)-K z\|^{2} \\
& =\|g(K z)\|^{2}-2\langle g(K z), K z\rangle+\|K z\|^{2} \\
& \leq\|K z\|^{2}-\langle g(K z), K z\rangle . \quad \text { (A.54) }
\end{aligned}
$$

We distinguish between two cases.

CASE 1: If $\|K z\| \leq \delta$, then, invoking the first inequality in $(\mathcal{F} .2)$, it follows from (A.54) yields that

$$
\|f(z)-K z\|^{2} \leq\|K z\|^{2}-\beta\|K z\|^{2}=(1-\beta)\|K z\|^{2} .
$$

Without loss of generality, we may assume that $\beta \in$ $(0,1)$, and so

$$
\begin{aligned}
\|f(z)-K z\| & \leq \sqrt{1-\beta}\|K\|\|z\| \\
& \leq\|K\|\|z\|-\alpha_{1}(\|z\|),
\end{aligned}
$$

for all $\alpha_{1} \in \mathcal{K}$ which satisfy $\alpha_{1}(s) \leq\|K\|(1-\sqrt{1-\beta}) s$ for all $s \geq 0$.

CASE 2: If $\|K z\| \geq \delta$, then the second inequality in $(\mathcal{F} .2)$ combined with (A.54) yields that

$$
\|f(z)-K z\|^{2} \leq\|K z\|^{2}-\gamma\|K z\| \leq(\|K z\|-\gamma / 2)^{2} .
$$

Without loss of generality, we may assume that $\gamma / 2 \leq$ $\delta \leq\|K z\|$ and, therefore,

$$
\begin{aligned}
\|f(z)-K z\| & \leq\|K z\|-\gamma / 2 \leq\|K\|\|z\|-\gamma / 2 \\
& \leq\|K\|\|z\|-\alpha_{2}(\|z\|),
\end{aligned}
$$

for all $\alpha_{2} \in \mathcal{K}$ which satisfy $\alpha(s) \leq \gamma / 2$ for all $s \geq 0$. Hence, in light of (A.54) and (A.55), taking $\alpha:=\min \left\{\alpha_{1}, \alpha_{2}\right\} \in \mathcal{K}$ ensures that $f$ satisfies (3.1), completing the proof.

In the following, for $\sigma \in \mathcal{S}^{m}$ and $l>0$, it is convenient to define $\sigma^{l}(z):=\sigma(l z)$ for all $z \in \mathbb{R}^{m}$. It is obvious that $\sigma^{l} \in \mathcal{S}^{m}$. To facilitate the proof of Proposition 4.4, we state a simple lemma.

Lemma A.4. Let $\sigma \in \mathcal{S}$. The following statements hold.

(1) If there exists $l>0$ such that

$$
\left(\sigma^{l}(s)\right)^{2} \leq s \sigma^{l}(s) \quad \forall s \in \mathbb{R},
$$

then, for any $k>0$

$$
\left(\sigma^{k}(s)\right)^{2} \leq(k / l) s \sigma^{k}(s) \quad \forall s \in \mathbb{R} .
$$

(2) Assume that there exist positive $l, b$ and $\delta$ such that

$$
s \sigma^{l}(s) \geq b s^{2} \quad \forall s \in \mathbb{R} \text { s.t. }|s| \leq \delta .
$$

Then, for any $k>0$,

$$
s \sigma^{k}(s) \geq(k / l) b s^{2} \quad \forall s \in \mathbb{R} \text { s.t. }|s| \leq(l / k) \delta .
$$


(3) For every $l>0$ and every $\varepsilon>0$, there exists $c>0$ such that

$$
s \sigma^{l}(s) \geq c|s| \quad \forall s \in \mathbb{R} \text { s.t. }|s| \geq \varepsilon .
$$

The straightforward proof of the above lemma is left to the reader.

Proof of Proposition 4.4. We consider the cases $m=$ 1 and $m>1$ separately.

CAse 1: $m=1$. Let $\sigma \in \mathcal{S}$. By the local Lipschitz property, there exists $L>0$ such that

$$
|\sigma(s)| \leq L|s| \quad \forall s \in \mathbb{R} \text { s.t. }|s| \leq\|\sigma\|_{L^{\infty}},
$$

and thus,

$$
(\sigma(s))^{2} \leq L s \sigma(s) \quad \forall s \in \mathbb{R} \text { s.t. }|s| \leq\|\sigma\|_{L^{\infty}} .
$$

Obviously, we may assume that $L \geq 1$, and so, $l:=$ $1 / L \leq 1$. Consequently, by (A.56),

$$
\begin{aligned}
&\left(\sigma^{l}(s)\right)^{2} \leq L(l s) \sigma(l s) \leq s \sigma^{l}(s) \\
& \forall s \in \mathbb{R} \text { s.t. }|s| \leq\|\sigma\|_{L^{\infty}} .
\end{aligned}
$$

Moreover,

$$
\begin{aligned}
\left(\sigma^{l}(s)\right)^{2} \leq\|\sigma\|_{L^{\infty}}\left|\sigma^{l}(s)\right| \leq s \sigma^{l}(s) \\
\forall s \in \mathbb{R} \text { s.t. }|s|>\|\sigma\|_{L^{\infty}} .
\end{aligned}
$$

Together with (A.57) this gives

$$
\left(\sigma^{l}(s)\right)^{2} \leq s \sigma^{l}(s) \quad \forall s \in \mathbb{R},
$$

showing that $\sigma^{l}$ satisfies $(\mathcal{F} .1)$.

Since $\liminf |s| \rightarrow \infty \sigma(s) / s>0$, there exist positive constants $\beta$ and $\delta$ such that

$$
\frac{\sigma^{l}(s)}{s}=l \frac{\sigma(l s)}{l s} \geq \beta \quad \forall s \in \mathbb{R} \text { s.t. }|s| \leq \delta,
$$

implying that

$$
s \sigma^{l}(s) \geq \beta s^{2} \quad \forall s \in \mathbb{R} \text { s.t. }|s| \leq \delta .
$$

Furthermore, statement (3) of Lemma A.4 guarantees the existence of a constant $\gamma>0$ such that

$$
s \sigma^{l}(s) \geq \gamma|s| \quad \forall s \in \mathbb{R} \text { s.t. }|s|>\delta .
$$

Together with (A.58) this shows that $\sigma^{l}$ satisfies $(\mathcal{F} .2)$. Consequently, $\sigma^{l} \in \mathcal{F}$.

CASE 2: $m>1$. Let $\sigma \in \mathcal{S}^{m}$. Then there exist $\sigma_{1}, \ldots, \sigma_{m} \in \mathcal{S}$ such that

$$
\sigma(z)=\left(\sigma_{1}\left(z_{1}\right), \ldots, \sigma_{m}\left(z_{m}\right)\right)^{T} \quad \forall z=\left(z_{1}, \ldots, z_{m}\right)^{T},
$$

where $z_{i}$ denotes the $i$-th component of $z$. By Case 1 , there exist positive constants $l_{i}, \beta_{i}$ and $\delta_{i}$ such that, for all $i \in \underline{m}:=\{1, \ldots, m\}$,

$$
\left(\sigma_{i}^{l_{i}}(s)\right)^{2} \leq s \sigma_{i}^{l_{i}}(s) \forall s \in \mathbb{R}
$$

and $s \sigma_{i}^{l_{i}}(s) \geq \beta_{i} s^{2} \forall s \in \mathbb{R}$ s.t. $|s| \leq \delta_{i}$.
We set

$$
l:=\min _{i \in \underline{m}} l_{i}, \quad \delta:=\min _{i \in \underline{m}} \delta_{i} \quad \text { and } \quad \beta:=\min _{i \in \underline{m}}\left(\frac{l}{l_{i}} \beta_{i}\right),
$$

and note that, $l \leq l_{i}, \delta \leq \delta_{i}$ and $\beta \leq \beta_{i}$ for all $i \in \underline{m}$. Furthermore, by statement (1) of Lemma A.4,

$$
\left(\sigma_{i}^{l}(s)\right)^{2} \leq s \sigma_{i}^{l}(s) \quad \forall s \in \mathbb{R}, \forall i \in \underline{m},
$$

and, by statement (2) of Lemma A.4,

$$
s \sigma_{i}^{l}(s) \geq \beta s^{2} \quad \forall s \in \mathbb{R} \text { s.t. }|s| \leq \delta, \forall i \in \underline{m} .
$$

Statement (3) of Lemma A.4 guarantees the existence of a constant $\eta>0$ such that, for all $i \in \underline{m}$

$$
s \sigma_{i}^{l}(s) \geq \eta|s| \quad \forall s \in \mathbb{R} \text { s.t. }|s| \geq \delta / \sqrt{m} .
$$

Appealing to (A.59), we obtain that

$$
\begin{aligned}
\left\|\sigma^{l}(z)\right\|^{2} & =\sum_{i=1}^{m}\left(\sigma_{i}^{l}\left(z_{i}\right)\right)^{2} \\
& \leq \sum_{i=1}^{m} z_{i} \sigma_{i}^{l}\left(z_{i}\right)=\left\langle\sigma^{l}(z), z\right\rangle \quad \forall z \in \mathbb{R}^{m},
\end{aligned}
$$

showing that $\sigma^{l}$ satisfies $(\mathcal{F} .1)$.

We proceed to establish that $\sigma^{l}$ also satisfies $(\mathcal{F} .2)$. To this end, we make use of (A.60) to conclude that

$$
\begin{gathered}
\left\langle\sigma^{l}(z), z\right\rangle=\sum_{i=1}^{m} z_{i} \sigma_{i}^{l}\left(z_{i}\right) \geq \beta \sum_{i=1}^{m} z_{i}^{2}=\beta\|z\|^{2} \\
\forall z \in \mathbb{R}^{m} \text { s.t. }\|z\| \leq \delta .
\end{gathered}
$$

Finally, by (A.61),

$$
\begin{gathered}
\left\langle\sigma^{l}(z), z\right\rangle=\sum_{i=1}^{m} z_{i} \sigma_{i}^{l}\left(z_{i}\right) \geq z_{j} \sigma_{j}\left(z_{j}\right) \geq \eta\left|z_{j}\right| \\
\forall z \in \mathbb{R}^{m} \text { s.t. }\|z\|>\delta
\end{gathered}
$$

where $\left|z_{j}\right|=\max _{i \in m}\left|z_{i}\right| \geq\|z\| / \sqrt{m}>\delta / \sqrt{m}$. Hence, setting $\gamma:=\eta / \sqrt{m}$, we arrive at

$\left\langle\sigma^{l}(z), z\right\rangle \geq \eta \max _{i \in \underline{m}}\left|z_{i}\right| \geq \gamma\|z\| \quad \forall z \in \mathbb{R}^{m}$ s.t. $\|z\|>\delta$.

Together with (A.62) this shows that $(\mathcal{F} .2)$ holds for $\sigma^{l}$. Consequently, $\sigma^{l}$ is in $\mathcal{F}$.

Proof of Theorem 5.1. For a proof of statement (1) see [28, Proposition 8.2.1]

Assume now that $\Delta(z)>0$ for all $z \in \mathbb{R}^{m}, z \neq 0$. Statement (2) follows from Theorem 2.3 and Proposition 3.10. To facilitate the proofs of statements (3)(6), define $\gamma: \mathbb{R}_{+} \rightarrow \mathbb{R}_{+}$by

$$
\gamma(s)=r s-\sup _{\|z\|=s}\|f(z)-K z\|=\inf _{\|z\|=s} \Delta(z) \quad \forall s \geq 0 .
$$

The function $\gamma$ is continuous, $\gamma(0)=0$ and $\gamma(s)>0$ for all $s>0$. Moreover, setting

$$
\beta(s):=\inf _{\tau \geq s} \gamma(\tau) \quad \forall s \geq 0,
$$


we note that $\beta(0)=0$ and $\beta: \mathbb{R}_{+} \rightarrow \mathbb{R}_{+}$is nondecreasing and continuous. It is clear that either $\beta(s)>0$ for all $s>0$ or $\beta(s) \equiv 0$, and we note that the former is equivalent to $\liminf _{\|z\| \rightarrow \infty} \Delta(z)>0$. Finally, defining $\alpha: \mathbb{R}_{+} \rightarrow \mathbb{R}_{+}$by $\alpha(s):=\left(1-e^{-s}\right) \beta(s)$ for all $s \geq 0$, it is clear that $\alpha(0)=0, \alpha$ is continuous and

$$
\alpha(s) \leq \beta(s) \leq \gamma(s) \leq \inf _{\|z\|=s} \Delta(z) \quad \forall s \geq 0 .
$$

Consequently, $\alpha(\|z\|) \leq \Delta(z)$ for all $z \in \mathbb{R}^{m}$ and thus,

$$
\|f(z)-K z\| \leq r\|z\|-\alpha(\|z\|) \quad \forall z \in \mathbb{R}^{m} .
$$

To prove statement (3), assume that $\liminf _{\|z\| \rightarrow \infty} \Delta(z)>0$. Then $\alpha$ is strictly increasing, whence is in $\mathcal{K}$, and statement (3) follows from (A.63) and Theorem 3.1.

We proceed to prove statement (4). By the radial unboundedness assumption, it is obvious that $\gamma(s) \rightarrow$ $\infty$ as $s \rightarrow \infty$, and so $\beta(s) \rightarrow \infty$ as $s \rightarrow \infty$, which in turn implies that $\alpha(s) \rightarrow \infty$ as $s \rightarrow \infty$, showing that $\alpha \in \mathcal{K}_{\infty}$. Statement (4) is now a consequence follows of (A.63) and Theorem 3.1.

Next, before we prove statement (5), we establish statement (6). By hypothesis, there exist $\delta_{1}>0$ and $0<\rho_{1}<\rho_{2}$ such that

$\frac{\Delta(z)}{\|z\|} \geq \delta_{1} \quad \forall z \in \mathbb{R}^{m}$ s.t. $0<\|z\|<\rho_{1}$ or $\|z\|>\rho_{2}$.

By continuity of $\Delta$ and the assumption that $\Delta(z)>0$ for all $z \in \mathbb{R}^{p}, z \neq 0$, there exists $\delta_{2}>0$ such that

$$
\frac{\Delta(z)}{\|z\|} \geq \delta_{2} \quad \forall z \in \mathbb{R}^{m} \text { s.t. } \rho_{1} \leq\|z\| \leq \rho_{2} .
$$

Hence, $\Delta(z) \geq \delta\|z\|$ for all $z \in \mathbb{R}^{p}$, where $\delta:=$ $\min \left\{\delta_{1}, \delta_{2}\right\}$. Therefore,

$$
\|f(z)-K z\| \leq(r-\delta)\|z\| \quad \forall z \in \mathbb{R}^{p},
$$

and it follows from [11, Theorem 4.1] that system (1.1) is exponentially ISS.

To prove statement (5), let $\Gamma>0$ be given. By statement (4), the Lur'e system (1.1) is ISS, and so, for all $x^{0} \in \mathbb{R}^{n}$ and $v \in L^{\infty}\left(\mathbb{R}_{+}, \mathbb{R}^{q}\right)$ such that $\left\|x^{0}\right\|+\|v\|_{L^{\infty}} \leq \Gamma$, there exists $\lambda>0$ such that the output $C x+D_{\mathrm{e}} v$ of (1.1) satisfies

$$
\left\|C x(t)+D_{\mathrm{e}} v(t)\right\| \leq \lambda \quad \forall t \geq 0 .
$$

Since $\liminf _{\|z\| \rightarrow 0}(\Delta(z) /\|z\|)>0$ and $\Delta(z)>0$ for $z \neq 0$, there exists $\delta>0$ such that

$$
\frac{\Delta(z)}{\|z\|} \geq \delta \quad \forall z \in \mathbb{R}^{m} \text { s.t. } 0<\|z\| \leq \lambda,
$$

and so, for all $z \in \mathbb{R}^{m}$ such that $0<\|z\| \leq \lambda$

$$
\|f(z)-K z\| \leq(r-\delta)\|z\| .
$$

Define the function $\tilde{f}: \mathbb{R}^{p} \rightarrow \mathbb{R}^{m}$ by

$$
\tilde{f}(z):= \begin{cases}f(z), & \|z\| \leq \lambda \\ f\left(\lambda \frac{z}{\|z\|}\right)+(\|z\|-\lambda) K \frac{z}{\|z\|}, & \|z\|>\lambda .\end{cases}
$$

Observe that $\tilde{f}$ is locally Lipschitz, and, for $z \in \mathbb{R}^{p}$ with $\|z\|>\lambda$, we have that

$$
\begin{aligned}
\|\tilde{f}(z)-K z\| & =\left\|f\left(\lambda \frac{z}{\|z\|}\right)-K \lambda \frac{z}{\|z\|}\right\| \\
& \leq(r-\delta) \lambda \leq(r-\delta)\|z\| .
\end{aligned}
$$

Together with (A.64) this yields

$$
\|\tilde{f}(z)-K z\| \leq(r-\delta)\|z\| \quad \forall z \in \mathbb{R}^{p},
$$

and consequently, by statement (6), the Lur'e system

$$
\dot{x}=A x+B \tilde{f}\left(C x+D_{\mathrm{e}} v\right)+B_{\mathrm{e}} v, \quad x(0)=x^{0},
$$

is exponentially ISS. Now, by construction, for all $x^{0} \in$ $\mathbb{R}^{n}$ and $v \in L^{\infty}\left(\mathbb{R}_{+}, \mathbb{R}^{q}\right)$ such that $\left\|x^{0}\right\|+\|v\|_{L^{\infty}} \leq \Gamma$, the solution $x:=x\left(\cdot ; x^{0}, v\right)$ of (1.1) satisfies $f(C x(t)+$ $\left.D_{\mathrm{e}} v(t)\right)=\tilde{f}\left(C x(t)+D_{\mathrm{e}} v(t)\right)$ for all $t \geq 0$, and thus it also solves (A.65), showing that (1.1) semi-globally exponentially ISS. The proof is complete.

\section{References}

[1] D. Angeli, E.D. Sontag \& Y. Wang. A characterization of integral input-to-state stability, IEEE Trans. Automat. Control, 45 (2000), 1082-1097.

[2] M. Arcak \& A. Teel. Input-to-state stability for a class of Lurie systems, Automatica, 38 (2002), 1945-1949.

[3] R. Azouit, A. Chaillet, Y. Chitour \& L. Greco. Strong iISS for a class of systems under saturated feedback, Automatica, 71 (2016), 272-280.

[4] A. Berman \& R.J. Plemmons. Nonnegative Matrices in the Mathematical Sciences. SIAM, Philadephia, 1994.

[5] A. Bill, C. Guiver, H. Logemann \& S. Townley. The converging-input converging-state property for Lur'e systems, Math. Control Signals Systems, 29:4 (2017), https://doi.org/10.1007/s00498-016-0184-3.

[6] A. Chaillet, D. Angeli \& H. Ito. Combining iISS and ISS with respect to small inputs: the strong iISS property, IEEE Trans. Automat. Control, 59 (2014), 2518-2524.

[7] R. Curtain \& H. Zwart. Stabilization of collocated systems by nonlinear boundary control, Systems \& Control Lett., 96 (2016), 11-14.

[8] S.N. Dashkovskiy, D.V. Efimov \& E.D. Sontag. Input-tostate stability and allied system properties, Autom. Remote Control, 72 (2011), 1579-1614.

[9] A.T. Fuller. In the large stability of relay and saturated control systems with linear controllers, Internat. J. Control, 15 (1977), 486-505.

[10] C. Guiver, H. Logemann \& M.R. Opmeer. Transfer functions of infinite-dimensional systems: positive realness and stabilization, Math. Control Signals Systems, 29 (2017), https://doi.org/10.1007/s00498-017-0203-z.

[11] C. Guiver, H. Logemann \& M.R. Opmeer. Infinitedimensional Lur'e systems: Input-to-state stability and convergence properties. SIAM J. Control Optimiz. $\mathbf{5 7}$ (2019), 334-365.

[12] C. Guiver, H. Logemann \& S. Townley. Low-gain integral control for multi-input multi-output linear systems with input nonlinearities, IEEE Trans. Automat. Control, 62 (2017), 4776-4783. 
[13] W. M. Haddad \& D. S. Bernstein. Explicit construction of quadratic Lyapunov functions for the small gain, positivity, circle, and Popov theorems and their application to robust stability. Part I: continuous-time theory. Int. J. Robust Nonlinear Control, 3 (1993), 313-339.

[14] W.M. Haddad \& V. Chellaboina. Nonlinear Dynamical Systems and Control: a Lyapunov-Based Approach. Princeton University Press, Princeton, 2008.

[15] D. Hinrichsen \& A. J. Pritchard. Real and complex stability radii: a survey, in D. Hinrichsen \& B. Mårtensson (eds.) Control of Uncertain Systems, pp. 119-162, Birkhäuser, Boston, 1990.

[16] D. Hinrichsen \& A. J. Pritchard. Destabilization by output feedback, Differ. Integral Eqn, 5 (1995), 357-386.

[17] D. Hinrichsen \& A.J. Pritchard. Mathematical Systems Theory I. Springer-Verlag, Berlin, 2005.

[18] D. Hinrichsen \& N.K. Son. Robust stability of positive continuous-time systems, Numer. Funct. Anal. Opt., 17 (1996), $649-659$.

[19] B. Jayawardhana, H. Logemann \& E.P. Ryan. Input-tostate stability of differential inclusions with applications to hysteretic and quantized feedback systems, SIAM J. Control Optimization, 48 (2009), 1031-1054.

[20] B. Jayawardhana, H. Logemann \& E.P. Ryan. The circle criterion and input-to-state stability: new perspectives on a classical result, IEEE Control Systems Mag., 31 (2011), $32-67$.

[21] C.M. Kellet. A compendium of comparison function results, Math. Control Signals Systems, 26 (2014), 339-374.

[22] H.K. Khalil. Nonlinear Systems. 3rd ed., Prentice-Hall, Upper Saddle River, 2002.

[23] Z. Lin \& A. Saberi. Semi-global exponential stabilization of linear systems subject to "input saturation" via linear feedbacks. Systems \& Control Lett., 21 (1993), 225-239.

[24] W. Liu, Y. Chitour \& E. Sontag. On finite-gain stabilizability of linear systems subject to input saturation, SIAM J. Control Optimiz., 34 (1996), 1190-1219.

[25] W. Q. Liu, V. Sreeram \& K.L. Teo. Model reduction for state-space symmetric systems, Systems \& Control Lett., 34 (1998), 209-215.

[26] H. Logemann \& E.P. Ryan. Ordinary Differential Equations. Springer, London, 2014.

[27] H. Logemann \& S. Townley. Low-gain control of uncertain regular linear systems, SIAM J. Contr. Optimiz., 35 (1997), 78-116.

[28] E. Sarkans. Absolute Stability and Input-to-State Stability of Lur'e Systems, PhD Thesis, University of Bath, UK,
2014.

[29] E. Sarkans \& H. Logemann. Input-to-state stability for Lur'e systems, Math. Control Signals Systems, 27 (2015), 439-465.

[30] E. Sarkans \& H. Logemann. Input-to-state stability of discrete-time Lur'e systems, SIAM J. Control Optim., 54 (2016), 1739-1768.

[31] E. Sarkans \& H. Logemann. Stability of higher-order discrete-time Lur'e systems, Linear Algebra and its Applications, 506 (2016), 183-211.

[32] T.I. Seidman \& H. Li. A note on stabilization with saturating feedback, Discrete and Continuous Dynamical Systems, 7 (2001), 319-328.

[33] M. Slemrod. Feedback stabilization of a linear control system in Hilbert space with an a priori bounded control, Math. Control, Signals Systems, 2 (1989), 265-285.

[34] E.D. Sontag. An algebraic approach to bounded controllability of linear systems, Internat. J. Control, 39 (1984), $181-188$.

[35] E.D. Sontag. Smooth stabilization implies coprime factorization, IEEE Trans. Automat. Control, 34 (1989), 435443.

[36] E.D. Sontag. Comments on integral variants of ISS, Systems \& Control Lett., 34 (1998), 93-100.

[37] E.D. Sontag. Input to state stability: basic concepts and results, in P. Nistri \& G. Stefani (eds.) Nonlinear and Optimal Control Theory, pp. 163-220, Springer Verlag, Berlin, 2006.

[38] E.D. Sontag \& H. Sussmann. Nonlinear output feedback design for linear systems with saturating controls, in Proc. 29th IEEE Conference on Decision and Control 1990, pp. 3414-3416.

[39] H. Sussmann, E. D. Sontag \& Y. Yang. A general result on the stabilization of linear systems using bounded controls, IEEE Trans. Automat. Control, 39 (1994), 2411-2425.

[40] A.E. Taylor. The norm of a real linear transformation in Minkowski space, L'enseignement Mathématique, 4 (1958), 101-107.

[41] M. Vidyasagar. Nonlinear Systems Analysis, 2nd edition, Prentice-Hall, Englewood Cliffs, NJ, 1993.

[42] C. Wang \& G. Weiss. The iISS property for globally asymptotically stable and passive nonlinear systems, IEEE Trans. Automat. Control, 53 (2008), 1947-1951.

[43] J. C. Willems. Analysis of Feedback Systems, The MIT Press, Cambridge, MA, 1971. 